\title{
A Mean Field Spin Glass with Short-Range Interactions
}

\author{
J. T. Chayes ${ }^{1, \star}$, L. Chayes ${ }^{1, \star \star}$, James P. Sethna ${ }^{1, \star \star \star}$, and D. J. Thouless ${ }^{2, \star \star \star \star}$ \\ ${ }^{1}$ Laboratory of Atomic and Solid State Physics, Cornell University, Ithaca, New York 14853, \\ USA \\ ${ }^{2}$ Department of Physics, University of Washington, Seattle, WA 98195, USA
}

\begin{abstract}
We formulate and study a spin glass model on the Bethe lattice. Appropriate boundary fields replace the traditional self-consistent methods; they give our model well-defined thermodynamic properties. We establish that there is a spin glass transition temperature above which the single-site magnetizations vanish, and below which the Edwards-Anderson order parameter is strictly positive. In a neighborhood below the transition temperature, we use bifurcation theory to establish the existence of a nontrivial distribution of single-site magnetizations. Two properties of this distribution are studied: the leading perturbative correction to the Gaussian scaling form at the transition, and the (nonperturbative) behavior of the tails.
\end{abstract}

\section{Table of Contents}

I. Introduction . . . . . . . . . . . . . . . . . . . . . . . . . . 42

Ia. History . . . . . . . . . . . . . . . . . . . . . . . . . 43

Ib. Organization and Main Results . . . . . . . . . . . . . . . . 46

II. Derivation of the Magnetic Recursion Relations . . . . . . . . . . . . . 49

IIa. Percolation and Deterministic Ferromagnets . . . . . . . . . . . . 50

IIb. Random Boundary Fields in the Ising Case . . . . . . . . . . . . 53

IIc. Half-Space Systems and the Isotropic Lattice by Quadrature . . . . . . 56

III. Analysis of Moments . . . . . . . . . . . . . . . . . . . . . . . 61

IIIa. Some Parting Remarks About Ferromagnetism . . . . . . . . . . . 62

IIIb. Location of the Spin Glass Critical Point . . . . . . . . . . . . . 64

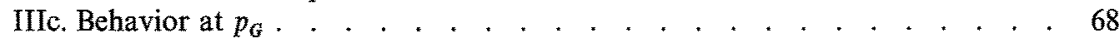

\footnotetext{
* Research supported by the NSF under Grant No. DMR-8314625

** Research supported by the DOE under Grant No. DE-AC02-83ER13044

$\star \star \star$ Research supported by the NSF under Grant No. DMR-8503544

$\star \star \star \star$ Research supported by the NSF under Grant No. DMR-8319301
} 
IV. Existence of a Spin Glass Near $p_{\mathbf{G}}$. . . . . . . . . . . . . . . . . . . . . . . . . . . 69

IVa. Preliminaries and Statement of Principal Results . . . . . . . . . . 70

IVb. The Linear Operators and Their Eigenfunctions . . . . . . . . . . 71

IVc. Existence of Nontrivial Solutions . . . . . . . . . . . . . . . . 73

IVd. Positivity of the Solutions . . . . . . . . . . . . . . . . . 79

V. Properties of the Solution . . . . . . . . . . . . . . . . . . . . . 81

Va. The Leading Non-Gaussian Correction. . . . . . . . . . . . . 82

Vb. Linear Stability and Perturbations in the Width Mode . . . . . . . . 84

Vc. Asymptotics at the Tails . . . . . . . . . . . . . . . . . . . 86

References. . . . . . . . . . . . . . . . . . . . . . . . 88

\section{Introduction}

Mean field theory, which approximates the behavior of a system by ignoring the effects of fluctuations, usually provides a good starting point for studying phase transitions. Sometimes fluctuations are irrelevant: systems in sufficiently many spatial dimensions or with sufficiently long-range interactions often have mean field critical behavior. Other times one can perturb about this limit to extract approximate information about the system of interest.

Developing useful mean field theories for disordered systems has been something of a challenge. The commonly studied Sherrington-Kirkpatrick (SK) model for spin grasses [1] starts with an infinite-range interaction - each spin interacts with every other spin. The solution of this model has taken many years to develop and understand; little progress has been made in extending the results to finite dimensions. Recently, one of the authors [2] has resurrected another approach to mean field theory for this system, and has studied the properties of spin glasses in infinite dimensions (on a Bethe lattice ${ }^{1}$ ).

Here we present some results characterizing the spin glass transition on the Bethe lattice with random boundary conditions. While this mean field limit may not have the same behavior as that of the Sherrington-Kirkpatrick model, the finite range of the interactions may illuminate better the extension to finite dimension, and the model is certainly more tractable.

We expect that this paper will have two somewhat disjoint readerships: mathematicians who are interested in learning about spin glasses and our methods for dealing with them, and physicists working on spin glasses who are interested in our results. This introduction first summarizes the history of the spin glass problem for those unfamiliar with it, and then summarizes our principal methods and conclusions.

\footnotetext{
${ }^{1}$ Previous work has distinguished carefully between the Bethe lattice and the Cayley tree. The Bethe lattice was considered an a priori infinite structure, while the Cayley tree is a thermodynamic limit of a finite tree. In many problems, the self-consistent solutions on the Bethe lattice were more physically relevant than those derived on the Cayley tree; however, the solutions depend sensitively on the chosen boundary conditions. In this paper, we develop a definite prescription for choosing boundary conditions and taking thermodynamic limits on the tree which in all cases seems to reproduce the Bethe lattice solutions. We therefore refer to our system as a Bethe lattice
} 


\section{Ia. History}

Spin glasses are typically formed by dilute magnetic impurities at random positions in a nonmagnetic, metallic host. The important degrees of freedom in these materials are the spins of impurity electrons. At very low concentrations and temperatures, the interactions of these spins with the Fermi sea of conduction electrons tend to "quench" them; this is the Kondo effect, which will not concern us here. At the concentrations of interest to us, the conduction electrons primarily mediate an effective interaction between the spins. The sharp edge of the Fermi surface leads to an effective interaction (the RKKY interaction) which oscillates in sign with distance and dies away relatively slowly.

These materials show a cusp in the magnetic susceptibility as a function of temperature. Above the cusp, the material behaves mostly as it would without interactions between the spins (as a paramagnet). Below this temperature, many indications suggest that the spins have frozen or locked into a disordered, glassy state; in particular, there is hysteresis on very long time scales, and relaxation with a broad distribution of frequencies. The cusp alone is suggestive of a transition; together with the onset of glassy behavior it seemed likely (although not certain) that a cooperative glass transition was occurring. Recent measurements of a divergence in the nonlinear susceptibility $[3,4]$ (signaling a diverging correlation length) have convinced most workers in the field that there is a glass transition in experimental (three-dimensional) spin glasses.

Edwards and Anderson [5] decided that the key feature of spin glasses is the random sign of the RKKY interaction, and that the dynamics of the conduction electrons, the random positions of the impurities, and the details of the strength and decay of the interactions with distance were unnecessary complications. Edwards and Anderson modelled each electron with an Ising $\operatorname{spin}^{2}$ : a two-state variable $s= \pm 1$. The effective interaction induced by the conduction electrons is represented by an alignment energy $2 J_{i j}$ between nearest-neighbor spins $s_{i}$ and $s_{j}$. The Hamiltonian is thus

$$
H=-\sum_{\langle i, j\rangle} J_{i j} s_{i} s_{j},
$$

where the sum is over all nearest-neighbor bonds $\langle i, j\rangle$ on a regular lattice of spins. The bond interactions are chosen at random ("quenched" disorder) from a symmetric distribution (e.g., $\pm J$ ). The interaction $J_{i j}$ is called ferromagnetic if it is positive (tending to align two spins); if it is negative it is antiferromagnetic. One central feature of this model is that it is frustrated: no arrangement of spins can satisfy all of the bonds. In particular, given a closed loop of nearest-neighbor spins on the lattice, with probability one half there will be an odd number of antiferromagnetic bonds on the loop. In such a loop, any configuration of spins must break one of the bonds. The Edwards-Anderson model is paramagnetic at high temperatures, but as the temperature is lowered there appears to be a transition below which there are many equilibrium states. Edwards and Anderson

\footnotetext{
${ }^{2}$ There are Heisenberg models of spin glasses, which in many cases describe the behavior more accurately. However, small crystalline effects break the spherical symmetry, and the behavior near the spin glass transition is thought to be described by the Ising version
} 
characterized the transition not by positivity of the magnetization (which should vanish for all positive temperatures), but instead by positivity of

$$
q \equiv \overline{\left\langle s_{i}\right\rangle^{2}},
$$

where the bar denotes a "quenched" average over realizations (see also [6]).

The central role of frustration in this model led many people to think about gauge invariance. Gauge transformations are relabeling schemes which leave the important physical properties invariant. In a spin glass, given any nonzero function $\tau_{j}$, the system described by spins $s_{j}$ and bonds $J_{i j}$ is equivalent (in the absence of an external field) to one with spins $s_{j}^{\prime}=\tau_{j} s_{j}$ and bonds $J_{i j}^{\prime}=\tau_{i}^{-1} J_{i j} \tau_{j}^{-1}$. (Typically, one chooses $\tau_{j}= \pm 1$.) The energy and frustrated loops are invariant under this transformation. Models with randomness but without frustration (e.g., the Mattis spin glass) can be formed by a random gauge transformation from an ordered system (e.g., a ferromagnet); these unfrustrated models are generally not taken seriously as models of spin glasses.

The first mean field model to provide useful information about the spin glass state [7] was an expansion in the inverse number of nearest neighbors $1 / Z$. The effects of loops are formally higher order in $1 / Z$, and this simplifies the problem to satisfying a set of self-consistent equations (the so-called TAP equations). Indeed, one derivation of the TAP equations starts with a spin glass on a "Bethe lattice" (see Fig. 1) - a lattice which branches indefinitely with branching ratio $Z$, on which the problem may be analyzed by the Bethe cluster method carried out to lowest order. Earlier work $[8,9]$ on spin glass models on the Bethe lattice is discussed in the next subsection. The identification of the Bethe lattice as an infinite-

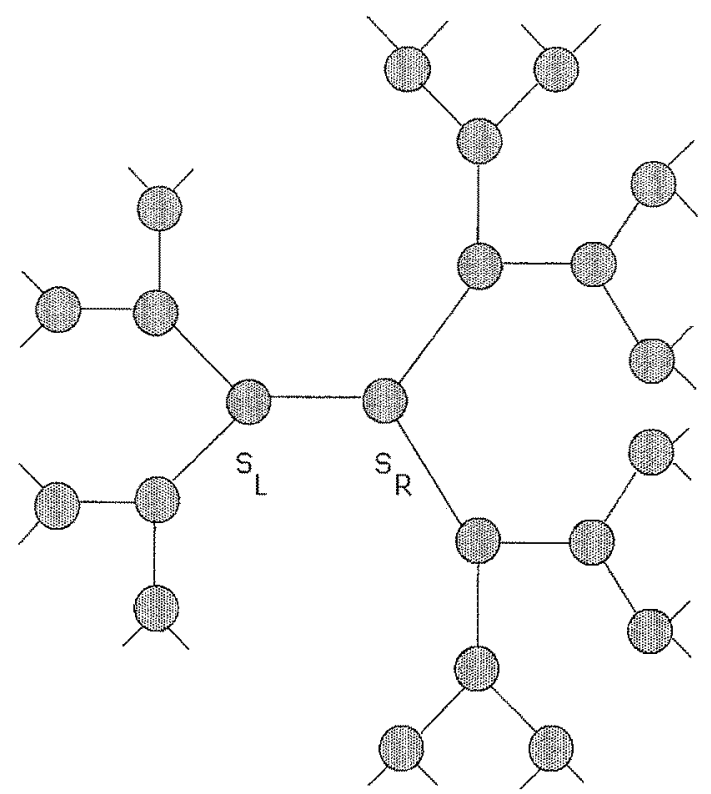

Fig. 1. Isotropic Bethe lattice with coordination number $Z=3$ 
dimensional limit is geometric. The Bethe lattice cannot be embedded in a finitedimensional lattice; alternatively, the number of sites that can be reached in an $N$-step walk ( $\sim N^{d}$ in a $d$-dimensional lattice) grows exponentially in $N$.

Solutions of the TAP equations gave good descriptions of numerical simulations of spin glasses, but interest in this model has waned. The TAP equations could only be approached numerically. Although algorithms for solving them were much more efficient than solving for the true spin glass equilibrium states, interest in numerical solutions of an approximate theory was limited.

There have also been doubts about the formulation of the spin glass problem on the Bethe lattice. On the one hand, with no loops where was the frustration? Any set of bonds can be satisfied by working step-by-step out from the center; a gauge transformation can push all of the disorder out to infinity. On the other hand, below the spin glass transition the behavior appeared to depend sensitively on boundary conditions. In formulating the problems on a tree structure, one must be careful to treat the boundary properly: if the forward branching ratio is $Z-1$, then all but $1 /(Z-1)$ of the spins are on the boundary! Generally speaking, one treats a large region of radius $R_{1}$ in the center of a tree of radius $R_{2}$, taking the boundary $R_{2}$ to infinity before taking the thermodynamic limit $R_{1} \rightarrow \infty$. The effects of the boundary conditions are supposed to die away with distance, in which case this order of limits generates the bulk behavior. However, the spin glass transition temperature is precisely the point at which the boundaries become important (the "shattered susceptibility" diverges [10]). We shall see that recent developments have improved matters: analytic methods are now possible, frustration is imposed by boundary conditions, and the effects of boundary conditions die away exponentially when treated properly.

The Sherrington-Kirkpatrick mean field theory for spin glasses, although proposed before TAP, gave useful low-temperature results only many years later. The Hamiltonian is of the Edwards-Anderson form (1.1) with a Gaussian distribution for $J_{i j}$, except that the interaction is infinite-ranged; each spin interacts with every other spin, and the sum extends over all pairs $\langle i, j\rangle$. After many false starts, it was pointed out that the low-temperature phase is characterized by "replica symmetry breaking" [11]; the first derivation of the solution to this model explicitly demonstrated the broken symmetry [12]. This forbiddingly formal solution was elucidated to a large extent by a series of papers explaining the nature of the order parameter [13-15], comparing it with numerical simulations [16], and giving information about the distances between different extremal states [17]. Shortly after the replica symmetry breaking solution, another approach incorporating relaxational dynamics was developed [18].

There are three important features of the solution to the infinite-range model. First, there are an infinite number of extremal states in the thermodynamic limit. Every time the system is cooled, a vastly different configuration of spins is observed; energy barriers between different states become infinite below the spin glass transition. Second, some extremal states are more alike than others. The overlap, $q_{\alpha x^{\prime}}$, between two extremal states $\alpha$ and $\alpha^{\prime}$, given by the average of $\left\langle s_{i} s_{i}^{\prime}\right\rangle_{\alpha \alpha^{\prime}}$ over all spins at finite temperature, is neither zero (as it would be in a paramagnetic phase); nor is its distribution concentrated on only a few values (as it would be in a 
ferromagnet or a Mattis spin glass with constant $\left.\left|J_{i j}\right|\right)$. Indeed, the probability distribution $W(q)$ for the overlap between extremal states is a nontrivial prediction of the theory. Thus the order parameter describing the mean field solution is not simply of the Edwards-Anderson form (1.2); it is a function $q(x)$, with $W(q)=d x / d q$. Third, the overlaps between extremal states group themselves into a tree-like hierarchy. Specifically, the topology is ultrametric; if the overlap is thought of as defining a distance on the space of the states, then each triangle is either isosceles with a base not longer than its sides or is equilateral. This can be used to organize the extremal states into a tree. Given an extremal state $\alpha_{0}$, put those extremal states whose overlap with $\alpha_{0}$ is greater than $q$ on a common branch; states on this branch will have overlaps no larger than $q$, and will be equidistant from any state not on the branch. We will see that the Bethe lattice also has a function $\varrho(x)$ as an order parameter; the tree structure of the Bethe lattice may relate to the hierarchy of extremal states of the Sherrington-Kirkpatrick model, but we have yet to make such a connection precise.

There has been some recent skepticism about the relevance of mean field theory to short-range spin glasses. The doubt is no longer that there is a spin glass transition; numerical simulations [19, 20], finite-size scaling calculations [21, 22] and the measurement of a divergence in the nonlinear susceptibility $[3,4]$ indicate that a freezing transition occurs in three dimensions. The question is whether the behavior in mean field is at all like that in short-range models in finite dimensions. On the one hand, the detailed behavior of the infinite-range model might be a pathology which has no analogue in finite dimensions. On the other hand, mean field theory misses important features of the dynamics. In finite dimensions, the flipping over of clusters of spins probably dominates the relaxation. Unfrustrated clusters have been shown to lead to an intermediate Griffiths phase between the spin glass and paramagnetic phase $[23,24]$; while relaxation in the paramagnetic phase is exponential, and the spin glass phase has long-range order in time, the Griffiths phase has nonexponential relaxation. Clusters of another sort (droplet excitations from the ground state) are the basis for a recent scaling description for the dynamics of spin glasses [25]. Clusters lose their meaning in mean field theory (in the sense that the surface to volume ratio of connected objects is of order one); without reinterpretation, none of these effects are thought to extend to infiniterange models or to infinite dimensions. While we have no reason to expect the Bethe lattice to be much better in these regards than the infinite-range model, it is so much simpler to handle that its implications for finite dimensions should be more apparent.

\section{Ib. Organization and Main Results}

The model we consider in this paper is the $\pm J$ Ising spin glass on the Bethe lattice with forward branching ratio two. Figure 1 shows the Bethe lattice. Each site, with Ising spin $s= \pm 1$, is connected to three others; each level of the tree has twice the number of sites of the previous level. The bonds have an interaction strength either $+J$ or $-J$, with equal probability. Henceforth in this paper, we will take $|J|=1$. The Hamiltonian is thus of the same form as the Edwards-Anderson Hamiltonian (1.1) described above:

$$
H=-\sum_{\langle i, j\rangle} J_{i j} s_{i} s_{j} .
$$


There is a long history of related work on random systems on the Bethe lattice. As mentioned above, there was very early work on random bond Bethe lattices, by groups in Tokyo [8] and Tohoku [9], which derived recursion relations and found a spin glass transition temperature. Bowman and Levin [26] examined the entropy of the spin glass state. More recently, the random field problem was studied by Bruinsma [27]. Finally, there is the work of [2], of which this work is the continuation.

We conclude this introduction with a brief "road map" of our results.

There are two important topics addressed in Sect. II: boundary conditions and recursion relations. The correct choice of boundary conditions is a key element in our approach. To emphasize this, we will first discuss the deterministic Potts systems on the Bethe lattice, where the correct (Bethe cluster, self-consistent) solution results from ferromagnetic boundary conditions, while free boundary conditions lead to spurious results. We then turn to the spin glass, where the spins at the boundary are taken to have some arbitrary but fixed configuration. Any such boundary condition transforms into random boundary fields under the gauge transformation which makes all bonds ferromagnetic; in the bulk of this paper, we assume that this gauge transformation has been performed. By fixing our boundary conditions, we have not only explicitly exhibited the frustration, but we have also set up a concrete statistical mechanics problem.

Working on the tree allows us to analyze the problem by a recursive method. Consider the half-space Bethe lattice with $n$ levels in Fig. 2. For any given set of bonds and any boundary condition, there is a magnetization $\mu_{0}=\left\langle s_{0}\right\rangle$ for the spin, $s_{0}$, at the base of the tree. Consider $t w o$ branches with base spins $s_{A}$ and $s_{B}$; one can calculate the magnetizations $\mu_{A}$ and $\mu_{B}$ that they would have had if they were disconnected from spin $s_{0}$. It so happens that $\mu_{0}$ can be written explicitly in terms of $\mu_{A}$ and $\mu_{B}$ :

$$
\mu_{0}=F\left(\mu_{A}, \mu_{B}\right)=p_{e}\left(\mu_{A}+\mu_{B}\right) /\left(1+p_{e}{ }^{2} \mu_{A} \mu_{B}\right),
$$

where $p_{e}=\tanh \left(1 / k_{B} T\right)$. That is, details about what happens on the rest of the tree are not important. According to the distribution of boundary fields, there will be a distribution, $d \varrho_{n}(\mu)$, of magnetizations for the $n^{\text {th }}$ level. The large- $n$ behavior of this distribution characterizes the spin glass. Since the two branches are uncorrelated, and since $\mu$ may be written in terms of $\mu_{A}$ and $\mu_{B}, \varrho_{n}$ may be written as a

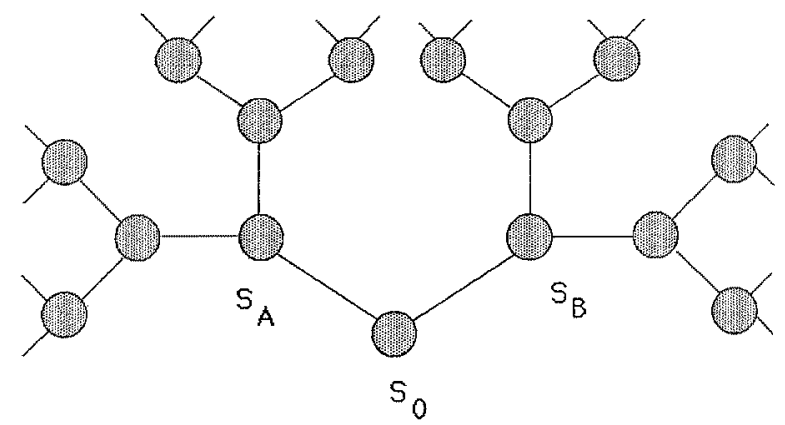

Fig. 2. A half-space Bethe lattice with forward branching ratio $Z-1=2$ 
convolution of $\varrho_{n-1}$ with itself; that is, formally,

$$
\varrho_{n}(\mu)=\int d \mu_{A} \int d \mu_{B} \varrho_{n-1}\left(\mu_{A}\right) \varrho_{n-1}\left(\mu_{B}\right) \delta\left(\mu-F\left(\mu_{A}, \mu_{B}\right)\right) .
$$

Finally, the full-space magnetization $\underline{\mu}_{L}$ of the spin $s_{L}$ of the isotropic Bethe lattice in Fig. 1 can be derived from the half-space magnetizations $\mu_{L}$ and $\mu_{R}$, where the latter are computed assuming the connecting bond is removed:

$$
\mu_{L}=\left(\mu_{L}+p_{e} \mu_{R}\right) /\left(1+p_{e} \mu_{L} \mu_{R}\right)
$$

The distribution of magnetizations of the isotropic tree can be derived from the half-space distributions by an expression along the lines of Eq. (1.4).

In Sect. III, we use a moment analysis of the recursion relations to establish some global features of the magnetization distribution as a function of temperature. The critical temperature corresponds to $p_{e}=p_{G} \equiv 1 / \sqrt{2}$. Above this temperature $\left(p_{e}<p_{G}\right)$, the single site magnetizations are globally attracted to zero. That is, any initial distribution of magnetizations converges exponentially fast to a point mass at zero. Below this temperature, we place upper and lower bounds on the Edwards-Anderson order parameter (the second moment of the distribution). Any (nonzero) distribution of boundary magnetizations will iterate into this band within a finite distance of the boundary (see Fig. 3). As a consequence of our bounds, the critical behavior of the Edwards-Anderson order parameter is given by

$$
q \sim\left|p-p_{G}\right|^{\beta_{G}} \quad \text { as } \quad p \downarrow p_{G}
$$

with $\beta_{G}=1$.

While the moment analysis guarantees that the low-temperature behavior is nontrivial, it was not obvious (at least to the authors) that it is described by a fixed distribution of magnetizations. Section IV temporarily abandons probabilistic methods, and uses functional analysis and bifurcation theory to prove the

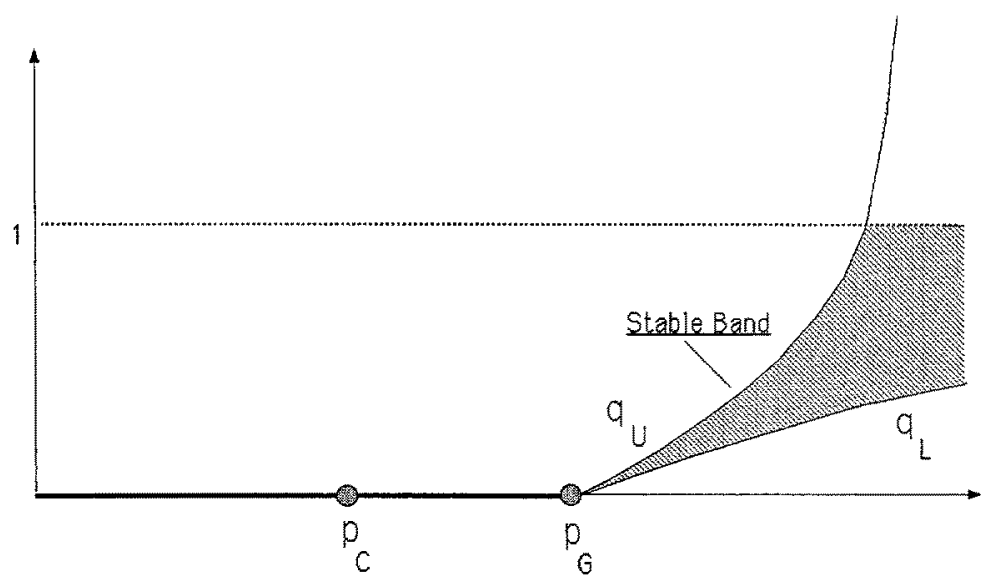

Fig. 3. The stable band. The shaded region, to the right of $p_{G} \equiv 1 / \sqrt{2}$, denotes allowed asymptotic values for the variance of any nontrivial symmetric initial distribution. Black line $\left(p \leqq p_{G}\right)$ indicates region of contraction for all symmetric distributions. The point $p_{c} \equiv 1 / 2$ is the critical point for the unfrustrated ferromagnet of corresponding strength 


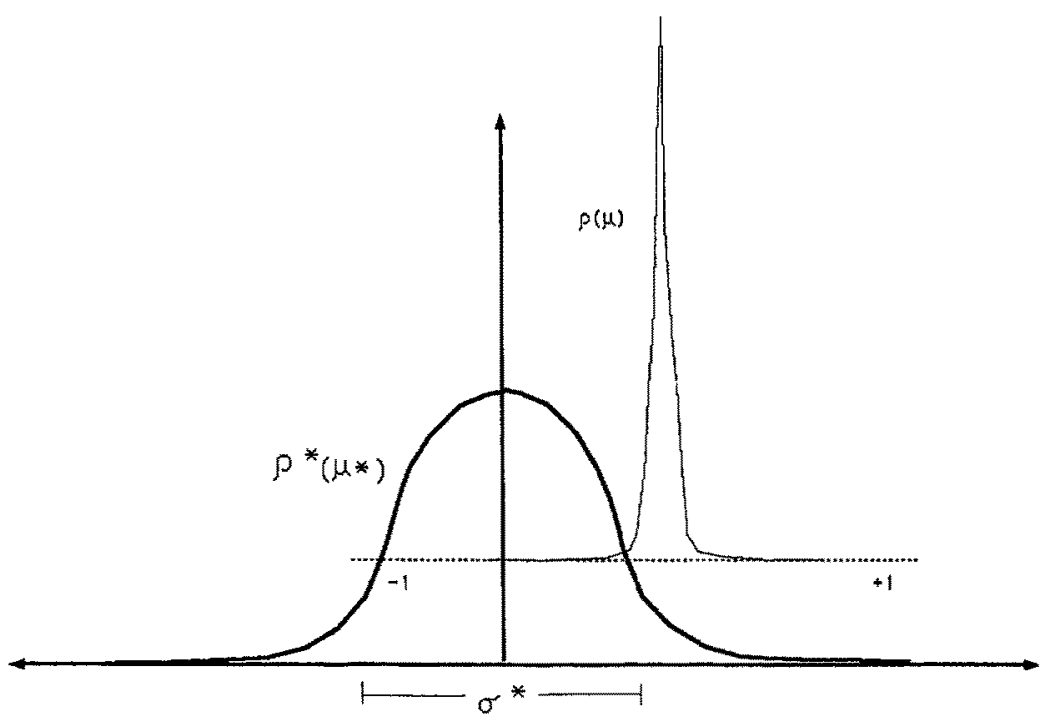

Fig. 4. The solution near $p_{G}$. Gaussian nature of the solution illustrated above. $\varrho(\mu)$ is the "actual" solution. The rescaled solution, $\varrho^{*}\left(\mu^{*}\right) \equiv \sqrt{\Delta} \varrho(\mu / \sqrt{\Delta})$, is Gaussian to lowest order. The rescaled width, $\sigma^{*}$, is a (computable) number of order unity

existence of a positive density as a fixed point to the recursion relation in a neighborhood above $p_{G}$. We call this fixed point function $\varrho_{\Delta}(\mu)$, where $\Delta \equiv p_{e}-p_{G}$. Since [by Eq. (1.6)] the second moment $q \approx$ (const) $\Delta$, there is a natural rescaling given by $\mu \rightarrow \mu / \sqrt{\Delta}$; in these rescaled variables, the distribution becomes Gaussian as $p \downarrow p_{G}$ (see Fig. 4).

In Sect. V, we briefly examine some of the properties of the spin glass solution $\varrho_{\Delta}(\mu)$. The first important question is whether the distribution is stable (an attractive fixed point). We cannot show that the distribution is globally attracting; indeed, in a technical sense we have not even shown linear stability for positive 4 . However, we have shown that the first order approximation to the functional derivative has all negative eigenvalues; this is a natural first step in a complete stability analysis. The second question we address is the shape of the distribution near the critical point. We solve for the function $\varrho_{A}$ to first order in $\Delta$, and find the leading nonGaussian correction. Finally, we consider the asymptotic behavior at the high and low ends of the magnetization distribution. Wherever $\varrho_{A}$ exists, we show the magnetization falls to zero as $\exp \left[-1 / \zeta^{c(p)}\right]$, where $\zeta$ measures the distance between $\mu$ and its maximum (or minimum) allowed value. In particular, we have upper and lower bounds on $c(p)$ of the form $(\log 2) / \log \{(1-p) /[($ const $) p]\} \mid$. These results are consistent with a picture of $\varrho_{\Delta}$ as an attractive, symmetric, Gaussian-like distribution with soft singular behavior at the tails.

\section{Derivation of the Magnetic Recursion Relations}

The distribution of single-site magnetizations contains much of the information on the behavior of a spin glass. The first step in obtaining the distribution is to derive a 
recursion relation between the magnetizations of certain sites. In this section, we give a first principles derivation of the magnetic recursion relation for the Ising spin glass on the Bethe lattice. Our main result is Eq. (2.27); except for this relation, the analysis in later sections is independent of the material presented here. Before the derivation of Eq. (2.27) (in Subsect. IIb), we will devote Subsect. IIa to a derivation of analogous results for certain deterministic spin systems.

In order that things be kept to a sensible level, we treat only the case of forward branching ratio 2 (i.e., $Z=3$ ). In addition, Subsects. IIa and Itb will be concerned only with a derivation of half-space quantities. Finally, in Subsect. IIc, we will show how a knowledge of these items (both in the deterministic and random systems) is sufficient to obtain the corresponding quantities in the isotropic lattice.

Subsection IIc also contains a detailed discussion of the significance of the recursion relations and their solutions for the construction of models in statistical mechanics. It is here that our approach differs fundamentally from the traditional one (which was used for deterministic systems in [28-30] and for random systems in $[8,9,26,27])$; rather than just studying the equations in their own right, we also show how they can be used to construct concrete infinite-volume systems whose single-site magnetizations are described by the solutions to the recursion relations.

\section{IIa. Percolation and Deterministic Ferromagnets}

The problem of percolation on the Bethe lattice is a canonical example of a branching process. As a problem in critical phenomena, it was thoroughly examined some time ago [31]. Here, we will be content with the most elementary derivation of the half-space infinite cluster density.

Consider a Bethe lattice with coordination number 3 on which bonds are occupied, independently, with homogeneous density $p \in[0,1]$. We define $P_{\infty}(p)$ to be the probability that, at bond density $p$, a given site is part of an infinite cluster in the half-space. Then

$$
P_{\infty}(p)=1-\left[1-p P_{\infty}(p)\right]^{2} .
$$

For $p \leqq 1 / 2$, the only solution is trivial, while if $p>1 / 2,(2.1)$ admits the "supercritical" solution

$$
P_{\infty}(p)=p^{-2}(2 p-1) .
$$

An elementary result from the theory of branching processes shows that for $p \geqq p_{c}=1 / 2,(2.2)$ is, in fact, the correct solution to (2.1).

It has been known since the work of Fortuin and Kasteleyn [32], that many magnetic systems can be treated as problems in interacting percolation. Consider an $n$-state Potts model given by the Hamiltonian

$$
H=-\sum_{\langle i, j\rangle}\left[n \delta\left(s_{i}, s_{j}\right)-1\right],
$$

where the spins $s_{i}$ take the values $1, \ldots, n$, and $\delta\left(s_{i}, s_{j}\right)=1$ if the two spins take the same value, and zero otherwise. At inverse temperature $\beta=1 / k_{B} T$, the FortuinKasteleyn (FK) representation describes the Potts model by an interacting percolation measure where the weight of a percolation configuration $\omega$ is given by

$$
W_{p, n}(\omega)=\mathscr{Z} B_{p}(\omega) n^{c(\omega)} \text {. }
$$


In the above, $p=p(n, \beta) \equiv 1-e^{-n \beta}, B_{p}(\omega)$ is the Bernoulli weight of $\omega$ at bond density $p, c(\omega)$ is the number of connected components of $\omega$ (counting each isolated site as a separate component), and $\mathscr{Z}$ is a normalization constant. As was noted in the original reference, all quantities of interest for statistical mechanics can be gleaned from a complete knowledge of the measures described by (2.4) when $n=2,3, \ldots$. For example, the constant $\mathscr{Z}$ is the partition function, and the "percolation" probability is identified with the spontaneous magnetization. On the other hand, for noninteger $n,(2.4)$ is well-defined as a random cluster model, which, like Bernoulli percolation, is capable of exhibiting a phase transition.

Of course, (2.4) only makes strict sense in a finite volume; in the study of phase transitions we must concern ourselves with the possible distributional limits of the measures described by (2.4) (i.e., the sense in which the thermodynamic limit is taken).

What is disturbing about (2.4) is that for a (finite piece of the half-space) Bethe lattice, the number of connected components $c(\omega)$ is simply related to the number of occupied bonds $b(\omega)$ and the total number of sites $S$ via

$$
c(\omega)=S-b(\omega) .
$$

It is easy to show that the measures defined from (2.4) with the rule (2.5) are none other than the ordinary percolation measures at effective parameter $p_{e}(p, n)$ $=p /[p+n(1-p)]$. Hence these systems undergo a transition as described by Eq. (2.2) at $p_{e}=1 / 2$. As has been discussed by numerous authors (see, e.g. [29]), this is annoyingly inconsistent with the results of the Bethe cluster method (or for that matter, the Curie-Weiss equations). In particular, the transition is always continuous and the magnetization exponent, $\beta$, takes on the value 1 (not $1 / 2$ if $n=2$ !) corresponding to percolation mean field behavior.

Our resolution of these difficulties (which is different from both the usual viewpoint - see e.g. $[28,29]$, and the alternative derivation of $[30])$ is that the noninteracting percolation measures are not the only possible distributional limits of the measures in (2.4). Indeed, tracing (2.4) together with rule (2.5), it is seen that (whenever $n=2,3, \ldots$ ) this corresponds to free boundary conditions for the spin system.

A popular way of inducing spontaneous magnetization in certain systems is via symmetry breaking at the boundary. (In principle, this should have the same effect on bulk properties as a vanishingly small magnetic field.) For the Ising model, the usual procedure is to set all spins at the boundary of the sample to +1 and, in the general $n$-state problem, we may set all boundary spins to the "first" spin state. In the random cluster picture, one can show that this corresponds to identifying all clusters touching the boundary as part of the same connected component, a procedure we call wiring of the boundary. This naturally suggests a set of welldefined problems for any positive value of $n$.

Remark. It is worth noting that for $n \geqq 1$, the random cluster measures enjoy the Harris-FKG $[33,34]$ property. As such, the finite volume measures with "wired boundary" are the extremal zero field conditional measures. Hence - assuming the infinite volume limit is taken along an ever increasing sequence - it is trivial to see that we arrive at the maximal state. In particular, this means that the resulting 
Fig. 5

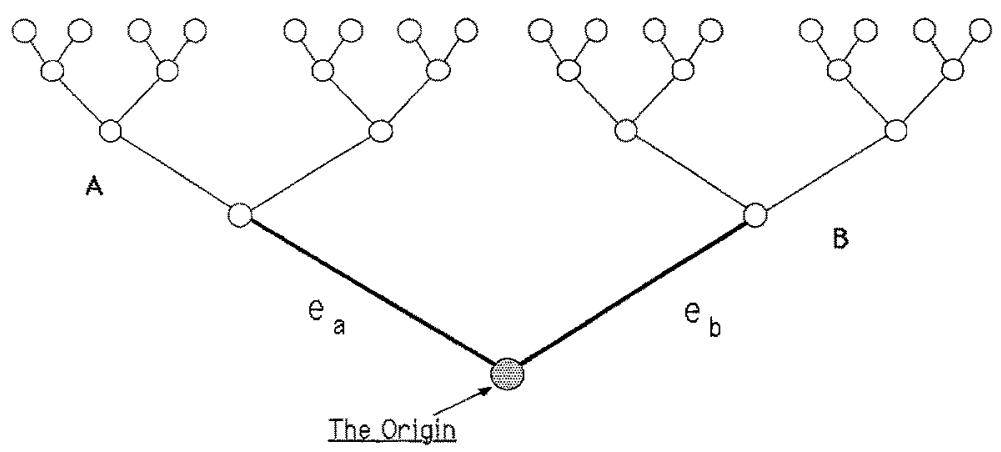

measure will FKG dominate the corresponding "free boundary" measures, so that if $n \geqq 1$, the percolation probabilities cannot be smaller than those dictated by (2.2). We will see that this indeed happens (rather dramatically) for all $n>1$.

The derivation of various percolation probabilities in the half-space is now quite straightforward. Let us consider two distinct lattices, denoted by $A$ and $B$, which are finite and "wired." We will couple these two lattices via the edges $e_{a}$ and $e_{b}$, as shown in Fig. 5. If $\omega$ is a configuration on the combined lattice, we may decompose $\omega$ according to

$$
\omega=\left(\omega_{A}, \omega_{B}, \zeta_{a b}\right),
$$

where $\omega_{A}$ is the restriction of $\omega$ to $A$, etc. Let us denote by $\chi_{a}$ and $\chi_{b}$ the indicators that the bonds $a$ and $b$, respectively, are "occupied." Finally, we will use the notation $W_{A}\left(\omega_{A}\right)$ for the weight of configuration $\omega_{A}$ in the uncoupled system, with a similar meaning assigned to $W_{B}\left(\omega_{B}\right)$. Explicitly, up to a constant (but volumedependent) factor,

$$
W_{A}\left(\omega_{A}\right)=B_{p_{e}}\left(\omega_{A}\right) n^{c^{*}\left(\omega_{A}\right)},
$$

where $c^{*}\left(\omega_{A}\right)$ is the number of clusters attached to the boundary of $A$.

Now the weights $W(\omega)$, which are given by a formula similar to $(2.7)$, will factor $W_{A}\left(\omega_{A}\right) W_{B}\left(\omega_{B}\right) B_{p_{e}}\left(\zeta_{a b}\right)$ unless the origin is connected to both the boundary of $A$ and that of $B$. In the latter case, we have overcounted the total number of connected components by one, and deserve an additional factor of $n$. Thus we have

$$
W(\omega)=W_{A}\left(\omega_{A}\right) W_{B}\left(\omega_{B}\right) B_{p_{e}}\left(\zeta_{a b}\right)\left[1+(n-1) \chi_{a}\left(\zeta_{a b}\right) \chi_{b}\left(\zeta_{a b}\right) \chi_{M_{A}}\left(\omega_{A}\right) \chi_{M_{B}}\left(\omega_{B}\right)\right],(2.8)
$$

where $M_{A}$ (respectively $M_{B}$ ) is the event that the "origin" of the $A$ (respectively $B$ ) lattice is in the connected component of the boundary.

Defining $\mathscr{Z} \equiv \sum_{\infty} W(\omega), \mathscr{Z}_{A} \equiv \sum_{\omega_{A}} W_{A}\left(\omega_{A}\right), m_{A} \equiv \mathscr{Z}_{A}{ }^{-1} \sum_{\omega_{A}} W_{A}\left(\omega_{A}\right) \chi_{M_{A}}\left(\omega_{A}\right)$, with corresponding definitions for the " $B$ " terms, we have

$$
\mathscr{Z}=\mathscr{Z}_{A} \mathscr{Z}_{B}\left(1+p_{e}{ }^{2}(n-1) m_{A} m_{B}\right) \text {. }
$$

The percolation probability (or magnetization) of the coupled system is given by

$$
\begin{aligned}
m \equiv & \mathscr{Z}^{-1} \sum_{\omega} W(\omega) \chi_{M}(\omega)=\mathscr{Z}^{-1} \sum_{\omega} W(\omega) \\
& \times\left[\chi_{a}\left(\zeta_{a b}\right) \chi_{M_{A}}\left(\omega_{A}\right)+\chi_{b}\left(\zeta_{a b}\right) \chi_{M_{B}}\left(\omega_{B}\right)-\chi_{a}\left(\zeta_{a b}\right) \chi_{M_{A}}\left(\omega_{A}\right) \chi_{b}\left(\zeta_{a b}\right) \chi_{M_{B}}\left(\omega_{B}\right)\right]
\end{aligned}
$$


by inclusion-exclusion. An easy computation yields

$$
m=p_{e}\left[m_{A}+m_{B}+p_{e}(n-2) m_{A} m_{B}\right] /\left[1+p_{e}^{2}(n-1) m_{A} m_{B}\right],
$$

so that in the infinite volume limit, the "wired" percolation probabilities must satisfy

$$
m^{(\infty)}=p_{e}\left[2 m^{(\infty)}+p_{e}(n-2)\left(m^{(\infty)}\right)^{2}\right] /\left[1+p_{e}{ }^{2}(n-1)\left(m^{(\infty)}\right)^{2}\right] .
$$

For $p_{e}<1 / 2$, the only solution of $(2.12)$ is the trivial solution $m^{(\infty)}=0$. However, to examine the solution at the critical point $p_{e}=1 / 2$ and beyond, we must distinguish three cases:

i) $1 \leqq n<2$

Here (2.12) implies the system has a continuous transition of the percolative type. The results near $p_{e}=1 / 2$ are

$$
m^{(\infty)}(p)=(n-2)^{-1}\left[\left(2 p_{e}-1\right) / p_{e}^{2}\right]\left[1+O\left(p_{e}^{-1 / 2}\right)\right] .
$$

ii) $n=2$

Again, the transition is continuous, but now we find

$$
m^{(\infty)}(p)=p_{e}{ }^{-1}\left(2 p_{e}-1\right)^{1 / 2}
$$

if $p_{e}>1 / 2$; we recover the usual Ising mean field behavior.

iii) $n>2$

In this case, no solution to (2.12) can yield a continuous transition for the obvious reason that $m^{(\infty)}$ would become negative. Elementary reasoning shows that a discontinuous transition occurs at $p_{e}=1 / 2$ with

$$
m^{(\infty)}(1 / 2)=(n-2) /(n-1) \text {. }
$$

Remarks. In all three cases, (2.12) yields the expected mean field behavior. Note that this is in contrast to the results obtained using free boundary conditions. However, the critical temperature is unaffected by boundary conditions, as one would expect.

It is also interesting to observe that when $1 \gg\left(p_{e}-1 / 2\right)^{1 / 2} \gg(2-n)>0$, the system has behavior qualitatively similar to the Ising $(n=2)$ case. Indeed, here one finds

$$
m^{(\infty)}\left(p_{e}\right)=p_{e}{ }^{-1}\left(2 p_{e}-1\right)^{1 / 2}\left[1+(1 / 4)(2-n) /\left(2 p_{e}-1\right)^{1 / 2}+O\left((2-n) /\left(2 p_{e}-1\right)^{1 / 2}\right)^{2}\right] .
$$

Equation (2.16) breaks down when $\left(p_{e}-1 / 2\right)^{1 / 2}$ is comparable to $(2-n)$, and the system exhibits a crossover to percolation-like behavior.

\section{IIb. Random Boundary Fields in the Ising Case}

In this subsection, we generalize our analysis to spin glass systems. Thus (2.3) is replaced by the random Hamiltonian:

$$
H\left(\left\{J_{i j}\right\}\right)=-\sum_{\langle i, j\rangle}\left[n J_{i j} \delta\left(s_{i}, s_{j}\right)-1\right],
$$


where the $J_{i j}$ take on the values +1 and -1 with equal probability. Since our principal concern is the Ising spin glass, we will henceforth restrict attention to the case $n=2$, so that up to additive constants (2.17) reduces to the Edwards-Anderson Hamiltonian (1.1).

As discussed in the introduction, for any realization $\left\{J_{i j}\right\}$ there exists a gauge transformation that reduces the system $(2.17)$ to one with purely ferromagnetic interactions, at the expense of introducing complications at the boundary. Whether nontrivial boundary conditions are actually induced is a consequence of the boundary conditions imposed on the untransformed system. Indeed, as has been demonstrated in the deterministic case, the boundary conditions must be chosen in accordance with the problem one wishes to address. In this light, it should be no surprise that a "spin glass" on the Bethe lattice with free boundary conditions corresponds to an (uninteresting) annealed problem, and is again described by the ordinary percolation results of Eq. (2.2). In order to describe a quenched spin glass, one should impose fixed (say + ) boundary conditions which become "unrecognizable" under the random gauge transformation. Thus a quenched spin glass on the Bethe lattice is equivalent to a ferromagnetic tree with random boundary conditions. We will sometimes refer to these induced boundary conditions as random boundary fields.

With nontrivial boundary fields (i.e., not free and not + ), a new feature enters the problem. Indeed, as is quickly revealed by examining the FK representation, a "cluster" of sites connected by occupied bonds represents a set of spin configurations in which all spins of the cluster are of the same type. Thus, for a finite piece of the Bethe lattice, $\mathscr{K}$, with fixed boundary condition $(\sigma \mid \partial \mathscr{K})$, any site in the connected component of the boundary is part of a cluster whose type is dictated by the condition at the boundary. In particular, the boundary condition $(\sigma \mid \partial \mathscr{K})$ implies that no two boundary spins of opposite type can be endpoints of the same bond cluster.

We will derive our recursion relation in much the same fashion as before (but specializing to the case $n=2$ ). However, here when we join two pieces of the lattice, we must take care to enforce the consistency condition: Two clusters which, under the directive of their respective boundary conditions, are deemed to be of opposite type must not be allowed to merge at the origin. This consistency condition is the essence of "frustration" on the Bethe lattice.

We again consider two pieces of lattice, $A$ and $B$, which now come equipped with some specified (but for the moment arbitrary) boundary condition. We define the event, $M_{A^{+}}$, that the origin of the $A$ system is attached to its boundary and terminates on a plus spin. The events $M_{A^{-}}, M_{B^{+}}$, and $M_{B^{-}}$are defined accordingly. All other symbols (e.g., $\mathscr{Z}_{A}, \mathscr{Z}_{B}$ etc.) have their previous meanings, only now it is understood that these objects are random variables, depending on the distribution of boundary spins. From the previous discussion, it should be clear that, for example, the events $M_{A^{+}}$and $M_{A^{-}}$are mutually exclusive (i.e., $M_{A^{+}} \cap M_{A^{-}}=\emptyset$ ).

Let us compute the $\mathscr{Z}$ obtained by coupling the $A$ and $B$ systems through the "origin." The weights for the coupled system are given by

$$
\begin{aligned}
W(\omega)= & W_{A}\left(\omega_{A}\right) W_{B}\left(\omega_{B}\right) B_{p_{e}}\left(\zeta_{a b}\right) \\
& \times\left[\left(1-\chi_{a}\right)\left(1-\chi_{b}\right)+\chi_{a}\left(1-\chi_{b}\right)+\chi_{b}\left(1-\chi_{a}\right)\right. \\
& +\chi_{a} \chi_{b}\left(1+\left(\chi_{M_{A^{+}}}+\chi_{M_{A^{-}}}\right)\left(\chi_{M_{B^{+}}}+\chi_{M_{B}}\right)\right] \chi_{++1--},
\end{aligned}
$$


where we have suppressed the arguments of the indicator functions. [To compare (2.18) with previous formulas, observe that here $n-1=1$.] In the above, $\chi_{++1--}$ is the newly required consistency condition which forbids a plus cluster attached to the boundary of one side to join with a minus cluster emanating from the boundary of the other. Explicitly

$$
\chi_{++1--}(\omega)=\left(1-\chi_{M_{A^{+}}}\left(\omega_{A}\right) \chi_{M_{B^{-}}}\left(\omega_{B}\right)\right)\left(1-\chi_{M_{A^{-}}}\left(\omega_{A}\right) \chi_{M_{B^{+}}}\left(\omega_{B}\right)\right)
$$

if $\chi_{a} \chi_{b}=1$, and is unity otherwise.

After a little algebra, one can show that the coefficient of $\chi_{a} \chi_{b}$ in Eq. (2.18) is given by

$$
1+\chi_{M_{A^{+}}} \chi_{M_{B^{+}}}+\chi_{M_{A^{-}}} \chi_{M_{B^{-}}}-\chi_{M_{A^{+}}} \chi_{M_{B^{-}}}-\chi_{M_{A^{-}}} \chi_{M_{B^{+}}} .
$$

In the derivation of the above fact, it is useful to note - as was implicitly assumed in (2.18) - that $\chi_{M_{A^{+}}} \chi_{M_{A^{-}}}=\chi_{M_{B_{B}}} \chi_{M_{B^{-}}}=0$ due to the internal consistency of the $A$ and $B$ lattices.

It is now easy to show that

$$
\mathscr{Z}=\mathscr{Z}_{A^{A}} \mathscr{Z}_{B}\left(1+p_{e}^{2}\left[m_{A^{+}} m_{B^{+}}+m_{A^{-}} m_{B^{-}}-m_{A^{+}} m_{B^{-}}-m_{A^{-}} m_{B^{+}}\right]\right),
$$

where

$$
m_{A^{+}}=\mathscr{Z}_{A}^{-1} \sum_{\omega_{A}} W_{A}\left(\omega_{A}\right) \chi_{M_{A^{+}}}\left(\omega_{A}\right)
$$

etc.

At this point, it is worth noting that a small, but important miracle occurs. All things considered, (2.21) is a relatively simple expression. However, if we define the "magnetization variables"

$$
\begin{aligned}
& \mu_{A}=m_{A^{+}}-m_{A^{-}}, \\
& \mu_{B}=m_{B^{+}}-m_{B^{-}},
\end{aligned}
$$

we have the still nicer expression

$$
\mathscr{Z}=\mathscr{Z}_{A} \mathscr{Z}_{B}\left(1+p_{e}{ }^{2} \mu_{A} \mu_{B}\right),
$$

indicating that, in this system, there are fewer pertinent degrees of freedom than one may have initially anticipated.

For the coupled system, we have

$$
\begin{aligned}
m_{+} & \equiv \mathscr{Z}^{-1} \sum_{\omega} W(\omega) \chi_{M^{+}}(\omega) \\
& =\mathscr{Z}^{-1} \sum_{\omega} W(\omega)\left(\chi_{a} \chi_{M_{A^{+}}}+\chi_{b} \chi_{M_{B^{+}}}-\chi_{a} \chi_{M_{A^{+}}} \chi_{b} \chi_{M_{B^{+}}}\right) \\
& =p_{e^{\mathscr{Z}}} \mathscr{Z}^{-1}\left(m_{A^{+}}+m_{B^{+}}-p_{e}\left(m_{A^{+}} m_{B^{-}}+m_{A^{-}} m_{B^{+}}\right)\right) .
\end{aligned}
$$

The expression for $m_{-}$is obtained from (2.25) by exchange of the symbols + and - Anticipating a (desirable) simplification, it is noticed that $\mu=m_{+}-m_{-}$is given by the formula

$$
\mu=p_{e}\left[\mu_{A}+\mu_{B}\right] /\left[1+p_{e}^{2} \mu_{A} \mu_{B}\right] .
$$

Any (Ising-type) system defined on the Bethe lattice in which the distribution of values of single-site magnetizations is stationary must be described by a 
(distributional) equation of the form (2.26). Explicitly, we consider a random variable $x$. Let $y$ and $z$ denote two independent random variables with identical distribution. Then we seek a solution to the distributional system

$$
x=p_{e}(y+z) /\left(1+p_{e}^{2} y z\right) \text {, }
$$

with

$$
x=y=\frac{\bar{d}}{d} z
$$

and

$$
y \mid z=\frac{d}{d} .
$$

\section{IIc. Half-Space Systems and the Isotropic Lattices by Quadrature}

Magnetic recursion relations similar to $(2.12)$ and $(2.27)$ have been derived by a number of authors in a variety of contexts $[28-30,8,9,26,27]$. Although our particular derivation in terms of the FK representation is new, it is similar to other derivations in that all have been motivated by considering systems described by the Hamilton functions (2.3) or (2.17) on the Bethe lattice. Given this motivation, one expects that solutions to such recursion relations should say something about the statistical mechanics of possible limiting systems described by these Hamiltonians; for this reason, the equations are certainly worth studying in their own right. However, the exact connection between the recursion relations and concrete problems in statistical mechanics is not immediately obvious, and seems to have been overlooked in previous work. Indeed, even in the deterministic case, the physical interpretation of a solution to the recursion relations is far from clear.

In this subsection, we examine the significance of the recursion relations and their solutions, and use this information to construct actual infinite-volume models whose magnetizations are described by these solutions (should they exist). Having constructed the infinite-volume half-space models, we then show that the half-space boundary fields can also be used to construct isotropic systems, and derive expressions for the magnetizations of the isotropic systems in terms of solutions to the half-space problems.

Consider first the deterministic case. Here, the solution of the recursion relation $(2.12)$ is just a number, $m^{(\infty)}$, which presumably corresponds to the magnetization at the origin in some infinite-volume system. This, however, gives us no clue as to what the magnetizations at the other sites should be in order that the magnetization at the origin be $m^{(\infty)}$. Before addressing this question directly, let us first consider the easier problem of constructing some infinite-volume system. In the deterministic case, it is always possible to do this by applying wired boundary conditions to an increasing sequence of finite-volume half-space lattices, and using the fact that for any (not necessarily integer) $n \geqq 1$, the FK random cluster measures so obtained are decreasing in the sense of FKG. Thus the sequence converges to a well-defined infinite-volume measure. Putting aside for the moment the question of whether the magnetization at the origin actually is $m^{(\infty)}$, it is obvious that the single-site magnetizations only increase on successive levels of the tree. (In fact, it is easy to show that the magnetizations strictly increase unless $m^{(\infty)} \equiv 0$.) Clearly 
then, it is not the single-site magnetizations themselves which are constant, and hence correspond to solutions of a recursion relation of the form (2.12).

What we must do is discover a quantity which is constant from site to site of the infinite-volume system constructed above. A little thought shows that if $x$ is located on the $k^{\text {th }}$ level from the origin, then the magnetization that $x$ would have had were it decoupled from the first $k-1$ levels of the tree is precisely the magnetization of the spin at the origin. Thus the quantity we are seeking - which is constant throughout the lattice - is the magnetization a spin would have in a hypothetical system in which that spin is decoupled from all spins on previous levels. Alternatively, in the FK representation (with wired boundary conditions) this quantity is a conditional probability; indeed, it is just the probability that a particular site is connected to infinity, provided that it is only connected to sites on higher levels.

With the above reasoning in mind, we will now construct the desired infinitevolume system. To do this, we use a procedure that is well-known in the theory of branching processes, and allows us to view (2.12) as an iterative map. Consider, then, an infinite sequence of $n$-state spin variables (for integer $n \geqq 2$ ): $s^{(1)}{ }_{0}, s^{(1)}, s_{1}^{(1)}{ }_{2}, \ldots$, which are independent, and acted on by identical external fields so as to produce some magnetization $m^{(1)}$ on each of the sites (see Fig. 6). Next, consider a second "wave" of spins $s^{(2)}{ }_{0}, s^{(2)}{ }_{1}, \ldots$, each coupled to a pair of spins of the first "wave" with coupling strength corresponding to $p_{e}: s^{(2)}{ }_{0}$ is coupled to $s^{(1)}{ }_{0}$ and $s^{(1)}{ }_{1}, \ldots, s^{(2)}{ }_{k}$ is coupled to $s^{(1)}{ }_{2 k}$ and $s^{(1)}{ }_{2 k+1}$, etc. It is clear that the magnetization of the second wave, $m^{(2)}$, is given by the right-hand side of Eq. (2.12) as a function of $m^{(1)}$, i.e.

$$
m^{(2)}=f_{p_{e}}\left(m^{(1)}\right) \equiv p_{e}\left[2 m^{(1)}+p_{e}(n-2)\left(m^{(1)}\right)^{2}\right] /\left[1+p_{e}{ }^{2}(n-1)\left(m^{(1)}\right)^{2}\right] .
$$

Now, we couple a third wave to the second in the same fashion, and so forth. In general, the magnetization of the $(k+1)^{\mathrm{st}}$ wave is determined by the magnetization of the $k^{\text {th }}$ wave via

$$
m^{(k+1)}=f_{p_{e}}\left(m^{(k)}\right)
$$

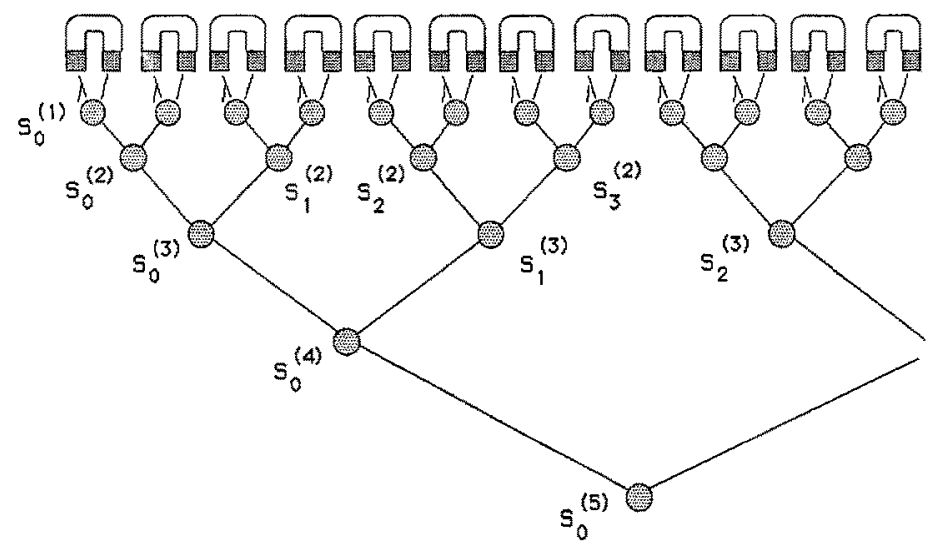

Fig. 6. A half-space problem 
As a result, we have a discrete time dynamical system whose behavior we cannot resist pausing to analyze. Plotting $f_{p_{e}}(x)$ as a function of $x$, we see that there are three potentially interesting points: $f_{p_{e}}(0)=0, f_{p_{e}}(1)$ $=p_{e}\left[2+p_{e}(n-2)\right] /\left[1+p_{e}{ }^{2}(n-1)\right]$, and, if $p_{e} \geqq 1 / 2$ (or $p_{e}>1 / 2$ when $n=2$ ), $f_{p_{e}}\left(m^{(\infty)}\right)=m^{(\infty)}$, where $m^{(\infty)}$ is the unique positive solution to Eq. (2.12).

A glance at the graphical construction in Fig. 7 indicates that any initial magnetization $m^{(1)} \in(0,1]$ converges to $m^{(\infty)}\left(p_{e}\right)$, and does so exponentially fast if $p_{e}>1 / 2$. For example, the wired boundary condition construction discussed earlier is just the case in which we begin with $m^{(1)}=1$. Furthermore, if the external field was chosen so as to produce $m^{(1)}=m^{(\infty)}\left(p_{e}\right)$, then for every $j, m^{(j)}$ will equal $m^{(\infty)}$. On the other hand, when $p_{e}<1 / 2$ (respectively $\leqq 1 / 2$ if $n=2$ ), the inevitable result is zero magnetization.

Even given the "backwards branching process" described above, it is not immediately obvious how to obtain meaningful thermodynamics on the tree, due to the large excess of surface. While this process ensures that the magnetization at the origin will be correct, it does not guarantee that the average (bulk) properties will settle down. Indeed, from the above discussion, we see that unless we start with $m^{(\mathbf{1})}=m^{(\infty)}\left(p_{e}\right)$, the large fraction of sites "near infinity" will not have magnetizations close to those of sites "deep inside the tree." The solution is to take the thermodynamic limit in two steps: Consider a large tree of radius $R_{1}$ inside a huge tree of radius $R_{2} \gg R_{1}$. From the backwards branching calculations, it is clear that the boundary of the large tree will have iterates $m^{\left(R_{2}-R_{1}\right)}$ which are close to $m^{(\infty)}$, and that these converge as $R_{2} \rightarrow \infty$. We may then take $R_{1} \rightarrow \infty$ to obtain wellbehaved thermodynamics.

One can also do the backwards branching process in the context of random systems. Here, however, the initial configuration of magnetizations should be distributed according to some random process. Although this is easily done by applying a random field to the first wave, for a possible future purpose, we will explicitly construct our random field as follows: Behind the first wave of spins, we place a zeroth wave of spins with alternating plus and minus signs: $s^{(0)}{ }_{0}=+1$, $s^{(0)}{ }_{1}=-1, s_{2}^{(0)}=+1, \ldots$. Let us emphasize that the $s^{(0)}{ }_{k}$ are fixed, and should not

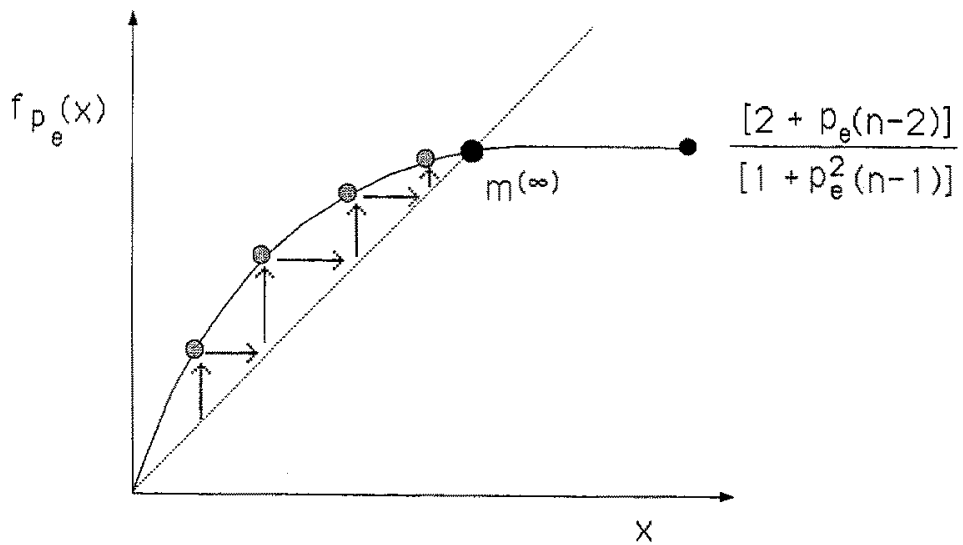

Fig. 7. Magnetization dynamics: the supercritical case 
be considered dynamical degrees of freedom. We now consider a sequence of pairs of bonds $\left.\left(J_{k^{+}}, J_{k^{-}}\right\} \mid k=0,1, \ldots\right)$ which couple the plus and minus spins, $s^{(0)}{ }_{2 k}$ and $s^{(0)}{ }_{2 k+1}$, to the $k^{\text {th }}$ spin of the first wave. Depending on the values of $\left\{J_{k^{+}}, J_{k^{-}}\right\}$, we can compute, as separate entities, the quantities $m^{+(1)}{ }_{k}, m^{-(1)}{ }_{k}$, and $\mu^{(1)}{ }_{k} \equiv m^{+(1)}{ }_{k}-m^{-(1)}{ }_{k}$. The pairs $\left\{J_{k^{+}}, J_{k^{-}}\right\}$are (identically and independently) assigned values according to some distribution, which in turn produces a distribution of magnetizations for the first wave. We then proceed, as in the previous example, to couple the second wave to the first with coupling strength $p_{e}$. The $\mu$ variables in the second wave will be distributed according to the right-hand side of Eq. (2.27) with $y$ and $z$ independently distributed in accord with the distribution of magnetizations of wave one. And so the process continues.

It should be emphasized that succeeding generations inherit their $\mu$ value exclusively from the $\mu$ value of the previous generation - regardless of how these values were produced. [For example, if $\mu$ is "small," this could be due to cancellation of $m^{+}$and $m^{-}$which are $O(1)$, or it could be that $m^{+}$and $m^{-}$are individually small.] This is exactly the reason we set the initial distribution up with "external spin pairs" rather than with an external field. The latter does not allow independent control over $m^{+(1)}{ }_{k}$ and $m^{-(1)}{ }_{k}$. At any rate, it is clear that should we find a solution to the system (2.27) and concoct (via the $\left\{J_{k^{+}}, J_{k^{-}}\right\}$) an initial distribution according to this solution, this distribution will persist in each successive wave. This does not, however, automatically imply that the joint or marginal distributions of the $m^{ \pm(j)}$ are themselves stationary.

Having established the half-space problems as bona fide models in their own right, it is worthwhile to make contact with the more popular "isotropic" models (see Fig. 1). Although the procedure is pretty much the same in the deterministic and random systems, we will do the calculations separately in order to make explicit yet another miracle.

Even in an isotropic system, the iterates $m^{(j)}$ are meaningful. In the language of spin systems, these quantities, of course, correspond to what the magnetization on the $j^{\text {th }}$ level (away from the boundary) would have been had we decoupled the spin from the back half of the lattice (or to conditional probabilities in the FK representation). From our construction above, it should be clear that, given the proper choice of boundary conditions, these conditional magnetizations are isotropic on the full lattice. It remains to be shown that the full magnetizations are determined exclusively by these half-space quantities.

The interpretation of the $m^{(j)}$ as conditional probabilities is the key to deriving the full-space quantities. Given two (finite) pieces of half-space lattice, $\mathscr{K}_{L}$ and $\mathscr{K}_{R}$, we would like to join them together to form a finite piece, $\mathscr{K}$, of the isotropic lattice. We will do this by attaching a bond, $b$, between the two base sites, $x_{L}$ and $x_{R}$, as indicated in Fig. 8.

Denote by $\mathscr{Z}_{L}$ and $\mathscr{Z}_{R}$ the partition functions of the two (independent) halfspace lattices. As before, we divide random cluster configurations, $\omega$, into $\left(\omega_{L}, \omega_{R}, \zeta_{b}\right)$, and denote by $M_{L}\left(M_{R}\right)$ the event that $x_{L}\left(x_{R}\right)$ is in the connected component of $\partial \mathscr{K}_{L}\left(\partial \mathscr{K}_{R}\right)$. Thus the left half-space magnetization at $x_{L}$ is

$$
m_{L} \equiv \mathscr{Z}_{L}^{-1} \sum_{\omega_{L}} W_{L}\left(\omega_{L}\right) \chi_{M_{L}}\left(\omega_{L}\right)
$$


Fig. 8

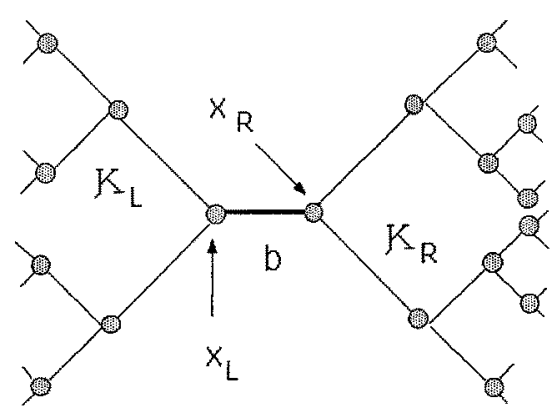

with a similar expression for $m_{R}$. Following the reasoning behind Eq. (2.8), we have

$$
W(\omega)=W_{L}\left(\omega_{L}\right) W_{R}\left(\omega_{R}\right) B_{p_{e}}\left(\zeta_{b}\right)\left[1+(n-1) \chi_{b}\left(\zeta_{b}\right) \chi_{M_{L}}\left(\omega_{L}\right) \chi_{M_{R}}\left(\omega_{R}\right)\right]
$$

which implies

$$
\mathscr{Z}=\mathscr{Z}_{L} \mathscr{Z}_{R}\left[1+p_{e}(n-1) m_{L} m_{R}\right] .
$$

The isotropic magnetization, $\underline{m}_{L}$, of the site $x_{L}$ in the full lattice $\mathscr{K}$ may be computed via (2.31) and the relation

$$
\chi_{M_{L}}=\chi_{M_{L}}+\left(1-\chi_{M_{L}}\right) \chi_{b} \chi_{M_{R}}
$$

where $M_{L}$ is the event that $x_{L}$ is connected to $\partial \mathscr{K}=\partial \mathscr{K}_{L} \cup \partial \mathscr{K}_{\mathbf{R}}$. Thus,

$$
m_{L}=\left[m_{L}+p_{e} m_{R}+(n-2) p_{e} m_{L} m_{R}\right] /\left[1+(n-1) p_{e} m_{L} m_{R}\right] \text {. }
$$

Evidently, if all the half-space magnetizations are given by $m^{(\infty)}$ [a solution to Eq. (2.12)], the isotropic magnetizations are found to be

$$
\underline{m}=m^{(\infty)}\left[1+p_{e}+(n-2) p_{e} m^{(\infty)}\right] /\left[1+(n-1) p_{e} m^{(\infty)}\right]
$$

The derivation for the random case follows in a similar fashion. We again consider two pieces of half-space lattice, $\mathscr{K}_{L}$ and $\mathscr{K}_{R}$, this time equipped with some (as yet unspecified) boundary conditions $\left(\sigma \mid \partial \mathscr{K}_{L}\right)\left[\right.$ respectively $\left.\left(\sigma \mid \partial \mathscr{K}_{R}\right)\right]$. Defining, as usual, the half-space plus and minus magnetizations, $m_{L^{+}}, m_{L^{-}}, m_{R^{+}}$, and $m_{R^{-}}$, a calculation similar to preceding ones gives the following expression for the weights:

$$
W(\omega)=W_{L}\left(\omega_{L}\right) W_{R}\left(\omega_{R}\right) B_{p_{e}}\left(\zeta_{b}\right)\left[1+\chi_{b}\left(\chi_{M_{L^{+}}}-\chi_{M_{L^{-}}}\right)\left(\chi_{M_{R^{+}}}-\chi_{M_{R^{-}}}\right)\right]
$$

and the total partition function:

$$
\mathscr{Z}=\mathscr{Z}_{L} \mathscr{Z}_{R}\left[1+p_{e} \mu_{L} \mu_{R}\right]
$$

where as before, $\mu_{L}=m_{L^{+}}-m_{L^{-}}$, etc. Observe that these are still the half-space quantities. Next let us compute the full-space "plus magnetization," $m_{L^{+}}$, which is given by the expression

$$
\underline{m}_{L^{+}}=\mathscr{Z}^{-1} \sum W(\omega) \chi_{M_{L^{+}}}(\omega)
$$

where is $\chi_{M_{L^{+}}}(\omega)$ given, explicitly, in terms of half-space quantities by the formula

$$
\chi_{M_{L^{+}}}=\chi_{M_{L^{+}}}+\left(1-\chi_{M_{L^{+}}}\right) \chi_{b} \chi_{M_{R^{+}}}
$$


Thus, we find

$$
\underline{m}_{L^{+}}=\left[m_{L^{+}}+p_{e} m_{R^{+}}-p_{e}\left(m_{L^{+}} m_{R^{-}}+m_{L^{-}} m_{R^{+}}\right)\right] /\left[1+p_{e} \mu_{L} \mu_{R}\right]
$$

with a corresponding expression for $\underline{m}_{L^{-}}$. Using the above formulas, the quantity $\mu_{L}$ admits the relatively simple expression in terms of the corresponding half-space quantities:

$$
\underline{\mu}_{L}=\left[\mu_{L}+p_{e} \mu_{R}\right] /\left[1+p_{e} \mu_{L} \mu_{R}\right]
$$

Assuming, then, that due to a clever choice of initial condition, we can produce distributions of $\left\{\mu_{j}\right\}$ which "settle down" as $j \rightarrow \infty$, one can compute the corresponding distribution of the full-space quantities. Indeed, one need only integrate the above formula with $\mu_{L}$ and $\mu_{R}$ independent and identically distributed according to the limiting distribution.

The full-space models can now be constructed via our knowledge of the halfspace quantities.

\section{Analysis of Moments}

In this section, we begin our analysis of the magnetic recursion relation (2.27) for the half-space random Ising system. As we have already learned, the half-space problems are well worth analyzing in their own right and, provided that a solution to the system (2.27) can be unearthed, the solution to the full-space problem can be constructed via (2.41). The main results of this section are summarized in Fig. 3 of the introduction; the bifurcation analysis of Sect. IV does not depend (explicitly) on any of these results.

Before we begin, it is worth simplifying the system (2.27) - and our notation somewhat. To this end, first observe that no limiting distribution can have support outside $\left[-m^{(\infty)},+m^{(\infty)}\right]$. (This follows from the FKG inequality.) Thus for $p>p_{c}=1 / 2$, we can scale our variables so that they live on a temperatureindependent interval. We define

$$
\begin{aligned}
& X \equiv x / m^{(\infty)}, \\
& Y \equiv y / m^{(\infty)}, \\
& Z \equiv z / m^{(\infty)},
\end{aligned}
$$

so that (2.27a) becomes $X=p_{e}[Y+Z] /\left[1+\left(p_{e} m^{(\infty)}\right)^{2} Y Z\right]$. For simplicity of future derivations, we will (by abuse of our earlier notation) drop the "effective" subscript on $p_{e}$ and simply use the variable $p$, which (since $n=2$ ) is related to the inverse temperature by

$$
p=\tanh \beta
$$

Finally, we also define ${ }^{3}$

$$
m(p) \equiv p m^{(\infty)}(p)=\left[(2 p-1)_{+}\right]^{1 / 2},
$$

\footnotetext{
${ }^{3} m(p)$ is actually the magnetization of a site attached to the origin of a half-space system by a bond of strength $p$
} 
where ( ) denotes the positive part of the function. In terms of these quantities, the distributional system (2.27) becomes

$$
\begin{aligned}
& X=p[Y+Z] /\left[1+m^{2} Y Z\right] \equiv F(Y, Z), \\
& X \underset{d}{=} Y=Z \\
& Y \mid Z=Y
\end{aligned}
$$

with $0 \leqq|X|,|Y|,|Z| \leqq 1$.

Ultimately, we would like to show that the random Ising system can support a spin glass phase. If such a phase exists, it must have a nontrivial symmetric distribution of magnetizations obeying the recursion relation (3.4); in particular, powers of (3.4) give coupled equations for the moments of such a distribution. It turns out to be impractical to attempt to establish the existence of a spin glass phase simply by studying moments of the distribution; at best, moments of low order give perturbative information on the magnetizations of such a phase, should it exist. For this system, one should be especially wary of purely perturbative statements, since if there is a spin glass phase, its magnetizations must be supported on a compact interval, and (in the final analysis) this type of behavior can only be achieved through the effects of nonperturbatively high moments.

In spite of the above considerations, a careful moment analysis enables one to make definite statements on the spin glass phase, should it exist, and suggests a starting point for a more complete treatment. This is the subject of this section.

Although it is not the purpose of this paper to discuss ferromagnetism, we begin this section with a brief analysis of nonsymmetric distributions.

Next, in Subsect. IIIb, we use a moment analysis to locate the critical point of the spin glass phase (again, should it exist). We do this by studying the second moment $q \equiv E\left(X^{2}\right)$, which is the (rescaled) Edwards-Anderson order parameter (1.2). The critical point is located in two steps: First, we show that there cannot be a spin glass phase (i.e. $q \equiv 0$ ) if $p \leqq 1 / \sqrt{2}$. Second, we show that, regardless of whether a distribution satisfying (3.4) exists, all but a finite number of the iterates of the second moment of any nondegenerate symmetric distribution lie within a strictly positive "stable band" whenever $p>1 / \sqrt{2}$. This allows us to identify $p_{G} \equiv 1 / \sqrt{2}$ as the spin glass transition point. Furthermore, as a corollary to the stable band analysis, we obtain the critical exponent for the Edwards-Anderson order parameter: $q(p) \sim\left|p-p_{G}\right|^{\beta_{G}}$ with $\beta_{G}=1$ as $p \downarrow p_{G}$. At this time, it is not clear whether this is indicative of the critical behavior (if any) of spin glasses in high dimension. It is, however, worth noting that $\beta_{G}=1$ also seems to hold in the infinite-range (SK) model of a spin glass.

Finally, in Subsect. IIIc, we examine an appropriately rescaled form of the magnetic recursion relation near $p_{G}$. The interesting property of this relation which constitutes the starting point of our analysis in Sect. IV - is that it can be solved exactly at $p_{G}$.

\section{IIIa. Some Parting Remarks About Ferromagnetism}

Before confining attention to symmetric distributions, it is worthwhile, at least as a warm-up, to spend a few paragraphs discussing (3.4) as a description of an honest 
ferromagnetic system. Clearly, below $p_{c}=1 / 2$ the only solution to the distributional system (3.4) is a point mass at the origin $X=0$, corresponding to the paramagnetic phase. However, above $p_{c}$, the system prefers the ferromagnetic state:

Proposition 3.1. Whenever $p>p_{c}=1 / 2$, the paramagnetic solution to (3.4) is unstable to ferromagnetism.

Proof. All that need be observed is that if the initial condition has a distribution which vanishes outside some small region, e.g. $\operatorname{Prob}[|Y|>\varepsilon]=0$, and, accordingly, has small first moment [say $E(Y)=\varepsilon k$ with $k=O(1)$ ], then the subsequent distribution given by (3.4) will have

$$
\varepsilon^{-1} E(X)=2 p k\left[1+O\left(\varepsilon^{2}\right)\right]>k
$$

for $\varepsilon$ sufficiently small. Thus, a point mass at the origin is an unstable distribution.

This result can be strengthened somewhat via:

Proposition 3.2. Whenever $p>1 / 2$, any nondegenerate initial distribution with no support on negative values has iterates that converge to the ferromagnetic state, which, in the language of (3.4) is a point mass at unity.

Proof. Let $X_{n}, Y_{n}, Z_{n}$ be random variables distributed according to the $n^{\text {th }}$ iterate of the initial distribution. It is not difficult to see that $\forall n, \operatorname{Prob}\left[X_{n}<0\right]=0$. Let us denote by $k_{n}$ and $q_{n}$ the first moment and variance of the $n^{\text {th }}$ distribution: $k_{n} \equiv E\left(X_{n}\right), q_{n} \equiv E\left(X_{n}^{2}\right)$. We have

$$
\begin{aligned}
X_{n+1}= & p\left[Y_{n}+Z_{n}\right] /\left[1+m^{2} Y_{n} Z_{n}\right]=p\left[Y_{n}+Z_{n}\right] /\left[1+m^{2}\right] \\
& +m^{2} p\left[Y_{n}+Z_{n}\right]\left[1-Y_{n} Z_{n}\right] /\left[1+m^{2} Y_{n} Z_{n}\right]\left[1+m^{2}\right] ;
\end{aligned}
$$

hence, since $1+m^{2} Y_{n} Z_{n} \leqq 1+m^{2}=2 p$,

$$
\begin{aligned}
k_{n+1} & \geqq 2 p k_{n} /\left[1+m^{2}\right]+2 m^{2} p k_{n}\left[1-q_{n}\right] /\left[1+m^{2}\right]^{2} \\
& =k_{n}\left[1+\left(m^{2} /\left(1+m^{2}\right)\right)\left(1-q_{n}\right)\right] .
\end{aligned}
$$

Thus, unless $k_{1} \equiv 0$, the $k_{n}$ form a monotone sequence which, after a little thought, is seen to converge to one.

Remark. We strongly suspect (but cannot yet prove) that the iterates of (3.4) are even more unstable to ferromagnetism than indicated by Propositions 3.1 and 3.2. This should not be disturbing to the reader since the random variables under discussion pertain to the gauge transformations of the actual magnetizations. When the magnetic interactions are equally likely to be of either sign, it is seen that the set of ferromagnetic boundary conditions (i.e., those with nonzero first moment) are of measure zero. Furthermore, it is easy to demonstrate that Eq. (3.4) maps symmetric distributions into symmetric distributions.

From a slightly different point of view, suppose we construct the spin glass problem iteratively (as in Subsect. IIc) - keeping track of the actual magnetizations. Then, at each stage of the iteration, the couplings must be selected 
according to some random process. By the time the second wave is coupled to the first, regardless of the initial distribution, the magnetizations in the second wave are described by a symmetric distribution (with probability one). Indeed, the distribution in the second wave is given by Eq. (3.4a) after symmetrization of the random variables on the right-hand side.

Thus, although the system is unstable to ferromagnetic perturbations, the quenched randomness provides an infinite restoring force towards the space of symmetric distributions. Henceforth, we will (safely?) restrict attention to this space.

\section{$I I I b$. Location of the Spin Glass Critical Point}

Here we examine the system (3.4) in the space of symmetrically distributed random variables.

For all values of $p$, one solution to (3.4) is a degenerate distribution: that is, $X=0$ with probability one. Our first result concerns the stability of this solution.

Proposition 3.3. The degenerate distribution is a stable solution to (3.4) if $2 p^{2}<1$, and is unstable if $2 p^{2}>1$.

Remark. In the borderline case, that is $2 p^{2}=1$, the degenerate distribution is also a stable solution, but this result follows from a slightly more detailed analysis.

Proof. As before, let us assume that $Y$ is not identically zero, but Prob $[Y>\varepsilon]=0$ and define $q_{0}=\varepsilon^{-1} E\left(Y^{2}\right)$. Squaring both sides of (3.4a), we have

$$
X^{2}=p\left[Y^{2}+Z^{2}+2 Y Z\right] /\left[1+m^{2} Y Z\right]^{2} .
$$

Defining $q_{1}=\varepsilon^{-1} E\left(X^{2}\right)$, we have [noting that $E(Y Z)=0$ by symmetry]

$$
q_{1}=2 p^{2} q_{0}\left(1+O\left(\varepsilon^{2}\right)\right) \text {. }
$$

Thus the second moment grows if $2 p^{2}>1$ and decays if $2 p^{2}<1$.

From the preceding analysis, it is already clear that there is a transition of some sort going on at $p=1 / \sqrt{2}$. Most of the rest of this section will be devoted to a detailed, nonperturbative treatment of the system (3.4), both above and below this point. Our principal results are:

A) global stability of the degenerate distribution if $2 p^{2} \leqq 1$,

B) existence of a "stable band" of allowed values of variances when $2 p^{2}>1$ (see Fig. 3).

Since the details of how $\mathbf{A}$ ) and $\mathbf{B}$ ) are established are not essential for the later analysis, Lemma 3.5 and Propositions 3.6-3.9 may be omitted without loss of continuity. For the benefit of the impatient reader, we will prove a weaker analogue of $\boldsymbol{A}$ ) which has the virtue that it can be "seen immediately."

Proposition 3.4. If $2 p^{2} /\left(1-m^{2}\right)^{2}<1$ (i.e. if $p<2-\sqrt{2}$ ), then the degenerate distribution is globally attracting.

Proof. Using the notation $X_{n}, Y_{n}, Z_{n}$ for the random variables distributed according to the $n^{\text {th }}$ iteration of (3.4a), we have, squaring both sides,

$$
X_{n+1}{ }^{2}=F^{2}\left(Y_{n}, Z\right) \underset{d}{=} p^{2}\left[Y_{n}^{2}+Z_{n}^{2}+2 Y_{n} Z_{n}\right] /\left[1+m^{2} Y_{n} Z_{n}\right]^{2} \text {. }
$$


However, $F^{2}(Y, Z) \leqq p^{2}\left[Y^{2}+Z^{2}+2 Y Z\right] /\left[1-m^{2}\right]^{2}$ whenever $|Y|,|Z| \leqq 1$, so that

$$
E\left[X_{n+1}^{2}\right]=E\left[F^{2}\left(Y_{n}, Z_{n}\right)\right] \leqq 2 p^{2} /\left(1-m^{2}\right)^{2} E\left[Y_{n}^{2}\right] \text {. }
$$

This means that if $q_{n}$ is the variance of $X_{n}$, then

$$
q_{n} \leqq\left[2 p^{2} /\left(1-m^{2}\right)^{2}\right]^{n},
$$

and hence is converging exponentially fast to zero.

The key to the proof of $\mathbf{A}$ ) and $\mathbf{B}$ ) is the following:

Lemma 3.5. If $m^{2} \leqq 2 / 3$ (or $\left.p \leqq 5 / 6\right)$, then $F^{2}(Y, Z)$ may be expressed in the form

$$
F^{2}(Y, Z)=p^{2}\left(Y^{2}+Z^{2}\right)+\Theta_{\text {odd }}(Y, Z)-R(Y, Z)
$$

with $R(Y, Z)$ pointwise positive, and $\Theta_{\text {odd }}(Y, Z)$ an odd function of $Y$ or $Z$.

Remark. The value $p=5 / 6$ is of no real significance, and could possibly be improved by a more detailed expansion of the type given below. (This would involve an attack on the fourth and higher moments.) Since the difficulty of these enterprises increases exponentially with ambition, we will be content with Lemma 3.5 as it stands.

Proof. We write the identity (valid whenever $\lambda \neq-1$ )

$$
1 /[1+\lambda]^{2}=1-2 \lambda+3 \lambda^{2}-4 \lambda^{3}+5 \lambda^{4} /[1+\lambda]^{2}+4 \lambda^{5} /[1+\lambda]^{2},
$$

which the reader is invited to verify. Using the above formula on $1 /\left[1+m^{2} Y Z\right]^{2}$, we have

$$
F^{2}(Y, Z)=p^{2}\left(Y^{2}+Z^{2}\right)+\Theta_{\text {odd }}(Y, Z)-R(Y, Z)
$$

with $\Theta_{\text {odd }}$ not worth computing and

$$
\begin{aligned}
R(Y, Z)= & 4 m^{2} p^{2} Y^{2} Z^{2}-3 m^{4} p^{2} Y^{2} Z^{2}\left(Y^{2}+Z^{2}\right) \\
& +8 m^{6} p^{2} Y^{4} Z^{4}-5 F^{2}(Y, Z) m^{8} Y^{4} Z^{4}-4 F^{2}(Y, Z) m^{10} Y^{5} Z^{5}
\end{aligned}
$$

Our job is to show that this is positive for the range of $p$ indicated.

Observe that since $F^{2}$ is neither even nor odd, we can conclude nothing about the parity of the last two terms without further expansion. For the range of $p$ under consideration, this turns out to be less efficient than the (universal) observation that $F^{2}(Y, Z)$ cannot exceed one.

Let us go to work on the first two terms:

$$
\begin{aligned}
4 m^{2} p^{2} Y^{2} Z^{2}-3 m^{4} p^{2} Y^{2} Z^{2}\left(Y^{2}+Z^{2}\right) & =4 m^{2} p^{2} Y^{2} Z^{2}\left[1-(3 / 4) m^{2}\left(Y^{2}+Z^{2}\right)\right] \\
& \geqq 4 m^{2} p^{2} Y^{2} Z^{2}\left[1-(3 / 2) m^{2}\right] \geqq 0
\end{aligned}
$$

if $m^{2} \leqq 2 / 3$.

That the remaining three terms add up to something positive follows from our universal observation. Indeed, rolling up our sleeves, we have

$$
\begin{aligned}
& 8 m^{6} p^{2} Y^{4} Z^{4}-5 F^{2}(Y, Z) m^{8} Y^{4} Z^{4}-4 F^{2}(Y, Z) m^{10} Y^{5} Z^{5} \\
& \quad=m^{6} Y^{4} Z^{4}\left(8 p^{2}-5 F^{2} m^{2}-4 F^{2} m^{4} Y Z\right) \\
& \quad \geqq m^{6} Y^{4} Z^{4}\left(2-m^{2}-2 m^{4}\right)
\end{aligned}
$$


where in the last step we have used $m^{2}=2 p-1$. The reader is invited to check that the final line in (3.17) is positive provided that $m^{2}<(1 / 4)\left[(17)^{1 / 2}-1\right]-$ which actually exceeds $2 / 3$ !

We are now prepared for:

Proposition 3.6. If $2 p^{2} \leqq 1$, the degenerate distribution is the unique, globally attractive solution of the system (3.4).

Proof. Starting from any initial distribution, we have, at the $n^{\text {th }}$ stage

$$
q_{n+1}=2 p^{2} q_{n}-E\left[R\left(Y_{n}, Z_{n}\right)\right],
$$

since $E\left[\Theta_{\text {odd }}(Y, Z)\right]=0$ by symmetry. If $2 p^{2}<1$, we may discard the (positive) remainder in (3.18), and watch exponential convergence of $q_{n}$ to zero. When $p=1 / \sqrt{2}$, we simply retain the first two terms in $R$, and use our bound (3.16). In this case, we have

$$
q_{n+1} \leqq q_{n}-r q_{n}^{2}
$$

with $r=4 m^{2} p^{2}\left[1-(3 / 2) m^{2}\right]$ evaluated at $p=1 / \sqrt{2}$. Here, the convergence may not be as fast, but the end result is the same.

When $2 p^{2}>1$, the existence of the upper limit is established in pretty much the same fashion:

Proposition 3.7. Suppose $5 / 6 \geqq p \geqq 1 / \sqrt{2}$. Then, given any initial distribution, all but a finite number of its iterates under (3.4) have variances bounded above by $q_{U}^{\prime} \equiv\left(2 p^{2}-1\right) /\left(4 p^{2} m^{2} r_{1}(p)\right)$ with $r_{1}(p)=1-(3 / 2) m^{2}$.

Remark. The above statement is obviously devoid of any significance when the upper bound exceeds unity. This, in fact, occurs even earlier than $p=5 / 6$. Nonetheless, we proceed in the spirit of the remark following the statement of Lemma 3.5.

Proof. Retaining only the first two terms of $R(Y, Z)$ and repeating the reasoning used in (3.19), we have the following inequality between the $n^{\text {th }}$ and $(n+1)^{\text {st }}$ variances

$$
q_{n+1} \leqq 2 p^{2} q_{n}-4 p^{2} m^{2} r_{1}(p) q_{n}^{2} .
$$

Let $q_{U}^{\prime}$ be defined as in the statement of the proposition. If $q_{n} \leqq q_{U}^{\prime}$, a worse bound than $(3.20)$ is

$$
q_{n+1} \leqq q_{U}^{\prime} .
$$

It is thus seen that when the initial distribution starts with a variance smaller than $q_{U}^{\prime}$, this condition persists for all $n$.

In the opposite case, i.e. when the initial variance starts above $q_{U}^{\prime}$, we may iterate (3.20) as though it were an identity to produce a sequence of uniform upper bounds on the $q_{n}$. [This follows from the fact that $q-4 p m^{2} r_{1}(p) q^{2}$ is increasing in $q$ for $0 \leqq q \leqq 1$.] This sequence of upper bounds converges (exponentially fast) to $q_{v}^{\prime}$. However, once one of the actual $q_{n}$ gets close enough to $q_{U}^{\prime}$, a slight improvement of the bound $R(Y, Z) \geqq 4 p m^{2} r_{1}(p) Y^{2} Z^{2}$ will push the succeeding $q$ 's strictly below $q_{U}^{\prime}$. 
For the lower piece of the band - which is the one of more importance when $p>1 / \sqrt{2}-$ the derivation follows with relative ease:

Proposition 3.8. Suppose $1 / \sqrt{2}<p \leqq 1$. Then, given any nondegenerate initial distribution, all but a finite number of its iterates under (3.4) have variances bounded below by $q_{L} \equiv\left(2 p^{2}-1\right) /\left(4 p^{2} m^{2}\right)$.

Proof. We first observe that the identity

$$
1 /(1+\lambda)^{2}=1-2 \lambda+\lambda^{2}(3+2 \lambda) /(1+\lambda)^{2}
$$

is valid provided $\lambda \neq-1$. Note that for $|\lambda|<1$, the third term is nonnegative. This gives

$$
F^{2}(Y, Z) \geqq 2 p^{2}\left(Y^{2}+Z^{2}\right)-4 p^{2} m^{2} Y^{2} Z^{2}+\text { "odd term." }
$$

Hence, we obtain the recursion inequality

$$
q_{n+1} \geqq 2 p^{2} q_{n}-4 p^{2} m^{2} q_{n}^{2} .
$$

Defining $q_{L}$ as above, it is seen that once any variance exceeds $q_{L}$, so do all the subsequent ones. This obviously handles the case in which we start out with a large enough $q$. On the other hand, when the initial $q$ is small (but nonzero by hypothesis), it is not difficult to show that (3.24) will provide a sequence of lower bounds which quickly places the actual $q_{n}$ within "striking distance" of $q_{L}$. The argument is completed along the lines of the preceding proposition.

Finally, it is worth showing that in the asymptotic regime, the upper and lower bounds actually coincide to first order.

Proposition 3.9. As $p \downarrow 1 / \sqrt{2}$, there is a $q_{U}$ satisfying the conclusions of Proposition 3.7 with the property that

$$
\lim _{p \downarrow 1 / \sqrt{2}} q_{v}(p) 4 p^{2} m^{2} /\left(2 p^{2}-1\right)=1 .
$$

Proof. Examining the expression (3.15) for $R(Y, Z)$, we easily obtain the recursion inequality

$$
q_{n+1} \leqq 2 p^{2} q_{n}-4 p^{2} m^{2} q_{n}^{2}+O\left(q_{n} t_{n}\right),
$$

where $t_{n}=E\left[X_{n}^{4}\right]$. Next, an uninspired examination of the fourth power of Eq. (3.4) permits the inequality

$$
t_{n+1} \leqq 2 p^{4} t_{n}+A(p) q_{n}^{2}
$$

where $A(p)$ is generously estimated by

$$
A(p) \leqq 65 p^{4} /\left(1-m^{2}\right)^{4} .
$$

Thus, eventually, $t_{n}<q_{U}^{\prime 2}\left[A(p) /\left(1-2 p^{4}\right)\right]$; i.e., the fourth moment is of the same order of magnitude as the square of the second. Equation (3.25) becomes

$$
q_{n+1} \leqq 2 p^{2} q_{n}-4 p^{2} m^{2} q_{n}^{2}+O\left(q_{U}^{\prime}{ }^{3}\right),
$$

from which the result is easily derived. 
We thus arrive at the picture (Fig. 3) described in the introduction. On the basis of this alone, we deserve the luxury of calling $p=1 / \sqrt{2}$ the spin glass point $p_{G}$. As an obvious consequence of the preceding analysis, we also have:

Corollary. If there is a spin glass (i.e. nondegenerate, symmetric) solution to the system (3.4) in a neighborhood of $p_{G}$, then the critical behavior of the EdwardsAnderson order parameter is given by

with $\beta_{G}=1$.

$$
q(p) \sim\left|p-p_{G}\right|^{\beta_{G}}
$$

\section{IIIc. Behavior at $p_{G}$}

As a consequence of Proposition 3.6, we already know that the distribution of magnetizations at $p_{G}$ is degenerate. This is not, however, the full story. Indeed, the upper and lower bounds of Propositions 3.7 and 3.8 suggest that, in the neighborhood of $p_{G}$, we should once again rescale our variables. Anticipating the order of magnitude of the asymptotic variances, it is natural to try

$$
\begin{aligned}
& X^{*}=[1 / \sqrt{\Delta}] X, \\
& Y^{*}=[1 / \sqrt{\Delta}] Y, \\
& Z^{*}=[1 / \sqrt{\Delta}] Z,
\end{aligned}
$$

with $\Delta \equiv p-p_{G}$. Under the above change of variables, (3.4a) becomes

$$
X^{*}=p\left[Y^{*}+Z^{*}\right] /\left[1+\Delta m^{2} Y^{*} Z^{*}\right]
$$

with $-[1 / \sqrt{\Delta}] \leqq X^{*}, Y^{*}, Z^{*} \leqq[1 / \sqrt{\Delta}]$.

Now as $p \downarrow p_{G},(3.30)$ becomes

$$
X^{*}=(1 / \sqrt{2})\left[Y^{*}+Z^{*}\right],
$$

which the reader will recognize as the addition law for normally distributed random variables. This provides us with a second reason for denoting the critical point by $p_{G}$.

The solution to (3.31), however, is of undetermined width. Thus, the "density"

$$
\varrho\left(X^{*}\right)=\left(\pi \sigma^{2}\right)^{-1 / 2} \exp \left[-X^{* 2} / \sigma^{2}\right]
$$

with any value of $\sigma$ might be deemed acceptable. That this is not the case has been demonstrated in Proposition 3.9. Here one sees (formally) that for $p$ near $p_{G}, q$ is given by

$$
q(p) \approx \Delta / p_{G} m^{2}\left(p_{G}\right),
$$

which implies

$$
\sigma^{2}=2 q(p) / \Delta \approx 2 / p_{G} m^{2}\left(p_{G}\right) .
$$

Such a result (which is not devoid of physical significance) will be encountered again when the question of existence of solutions to (3.4) is addressed. 


\section{Existence of a Spin Glass Near $p_{G}$}

In this section, we establish our principal result: namely, that the random Ising system on the Bethe lattice supports a spin glass phase with a nontrivial symmetric distribution of magnetizations, $\varrho_{\Delta}$, which is a fixed point of the integral equation (1.4). As we will see, the spin glass magnetizations are distributed according to a density which bifurcates from the trivial delta function solution at $p_{G}$. That the solution is a density excludes certain pathological behaviors (e.g., $\varrho_{A}$ can have nothing worse than integrable singularities).

Our proof begins with the observation made at the end of the previous section. There it was noted, at least formally, that if we rescaled the magnetization variables by $1 / \sqrt{\Delta}$ with $\Delta \equiv p-p_{G}$, then at $p_{G}$ the recursion relation reduces to the addition law for Gaussian random variables. Of course, since this scaling is singular at $p_{G}$, we have no guarantee that the Gaussians are actually related to the problem at hand. Nevertheless, together with the instability of the delta function solution for $p>p_{G}$, this rescaling suggests that a new solution may grow from one of the Gaussians.

A natural approach to the problem is to attempt a rescaled moment analysis by calculating corrections in $\Delta$ to the Gaussian addition law. If one does this, one finds that the $2 n^{\text {th }}$ moment is described by an equation which to leading order in $\Delta$ has terms involving all previous (even) moments. There are several difficulties with this program. First, it seems that the coefficients of such a perturbation theory grow extremely rapidly with $n$. Second, a detailed analysis (see Sect. Vc) indicates that nonperturbative effects are significant in this system.

For the above reasons, we have approached the question of existence of a spin glass state as a problem in bifurcation theory; in other words, we are going after a function which completely describes the distribution of single site magnetizations, rather than simply attempting to calculate its moments. To do this, we (temporarily) abandon the random variable equation (3.4), and look instead at an integral equation describing the distribution $\varrho_{\Delta}(x)$ of these random variables:

$$
\varrho_{\Delta}(x)=\int_{-1}^{+1} \varrho_{\Delta}(y) \varrho_{\Delta}(z) \delta(x-F(y, z)) d y d z,
$$

where $-1 \leqq x, y, z \leqq 1$, and

$$
F(y, z)=p[y+z] /\left[1+m^{2} y z\right]
$$

as before. Now despite the fact that the right-hand side of (4.1) is not well-defined for a general density $\varrho_{4}$, it still can be interpreted by means of the usual formulas. Since this leads to a rather cumbersome expression, Eq. (4.1) will be written properly for the first and last time:

$$
\varrho_{\Delta}(x)=p \int_{-1}^{+1} \varrho_{\Delta}(y) \varrho_{\Delta}\left([x-p y] /\left[p-m^{2} x y\right]\right)\left(\left[1-m^{2} y\right] /\left[p-m^{2} x y\right]^{2}\right) d y .
$$

Henceforth, when we use the informal notation of (4.1), it will be understood in the sense of $\left(4.1^{\prime}\right)$.

Our principal result is that for $p$ sufficiently near $p_{G}$ (i.e., $\Delta>0$ sufficiently small), there is a unique density $\varrho_{\Delta}$ satisfying Eq. (4.1), and hence describing the 
single site magnetizations of a spin glass. As $\Delta \downarrow 0$, the solution collapses to a delta function - which is a Gaussian in the rescaled variables - with a width vanishing linearly in $\Delta$.

The solution is also somewhat interesting from the viewpoint of bifurcation theory. First, being a density, it lives in a different space than the delta function solution from which it bifurcates. Second, as we have already seen, the system has "too many solutions" at $p_{G}-$ a Gaussian of any width satisfies the rescaled form of (4.1) when $\Delta=0$. This is indicative of a scale invariance symmetry which is broken at nonzero $\Delta$; it is precisely the breaking of this symmetry by nonlinearities that picks out the Gaussian of the "correct width." Third, the fact that (4.1) is an integral (rather than a differential) equation means that it has certain "smoothing properties." As a consequence, despite the fact that the unperturbed (rescaled) equation is defined on a noncompact space, the linear operator has a discrete spectrum.

The outline of this section is as follows: In Subsect. IVa, we formulate a decompactified version of the rescaled problem and state our main theorem. In Subsect. IVb, we study the linear operator of the decompactified problem, and compute all of its eigenvalues and eigenfunctions at $p_{G}$. Although the principal purpose of this section is to prove an (abstract) existence theorem, these eigenfunctions are of some independent interest. Indeed, once we have established the existence of a spin glass solution, these are the functions which best describe perturbative corrections to the Gaussian for $p>p_{G}$. The existence of a nontrivial solution is established in Subsect. IVc, and positivity of the solution is shown in Subsect. IVd.

\section{IVa. Preliminaries and Statement of Principal Results}

The purpose of our analysis is to find a positive function $\varrho_{\Delta} \in L^{1}([-1,+1])$ which is a solution to the integral equation (4.1). Our methods include use of the implicit function theorem, together with "exchange of stability" arguments. For this reason, it is convenient for the "known solution" [i.e., the Gaussian(s) at $p=p_{G}$ ] to live on the same space as the purported family of new solutions. Clearly $L^{1}([-1,+1])$ is a disaster! An obvious remedy is to use the rescaled intervals $[-1 / \sqrt{\Delta},+1 / \sqrt{\Delta}]$, but this suffers the annoying habit of changing - albeit continuously - with the parameter $\Delta$. Our resolution of these difficulties (which is by no means the only one) is to always work on $L^{1}(\mathbb{R})$. This will require some preliminary legwork that the authors feel is well worth the trouble.

Definition. Let $\Phi:[-1,+1] \rightarrow \mathbb{R}$ be a $C^{\infty}$, invertible, symmetric function which is the identity in a neighborhood of the origin. We define $\Phi_{\Delta}(x)=\Phi(x \sqrt{\Delta})$, so that $\Phi_{\Delta}:[-1 / \sqrt{\Delta},+1 / \sqrt{\Delta}] \rightarrow \mathbb{R}$ goes continuously to the identity as $\Delta$ tends to zero. The fixed choice of $\Phi$ (or $\Phi_{A}$ ) induces a compatible map between $L^{1}(\mathbb{R})$ and $L^{1}([-1,+1])$ :

$$
\varphi: L^{1}(\mathbb{R}) \rightarrow L^{1}([-1,+1])
$$

according to

$$
\varphi_{f}(x)=f(\Phi(x)) \Phi^{\prime}(x) \text { a.e. }
$$


with a similar definition for $\varphi_{A ; f}(x)$. Observe that the factor $\Phi^{\prime}(x)$ [respectively $\left.\Phi_{\Delta}^{\prime}(x)\right]$ ensures preservation of the norm under these induced maps.

We will be concerned with the decompactified version of (4.1). Let us therefore start by defining the family of nonlinear operators $\mathscr{F}_{4}: L^{1}(\mathbb{R}) \rightarrow L^{1}(\mathbb{R})$, given by

$$
\mathscr{F}_{\Delta}(f)=\varphi_{A}{ }^{-1}\left(\mathscr{H}_{\Delta}\left(\varphi_{A ; f}\right)\right) \text {, }
$$

where $\mathscr{H}_{\Delta}$ is the rescaled (or "stretched") version of the right-hand side of (4.1):

$$
\mathscr{H}_{\Delta}(h)[x]=\int_{-1 / \sqrt{\Delta}}^{+1 / \sqrt{\Delta}} h(y) h(z) \delta\left(x-F_{A}(y, z)\right) d y d z
$$

and $F_{A}(y, z)$ is determined from Eq. (3.30):

$$
F_{\Delta}(y, z)=p[y+z] /\left(1+\Delta m^{2} y z\right]
$$

with $-1 / \sqrt{\Delta} \leqq x, y, z \leqq 1 / \sqrt{\Delta}$. Of course, $\mathscr{F}_{\Delta}$ can be defined all at once by the single expression

$$
\mathscr{F}_{\Delta}(f)[\xi]=\int_{-1 / \sqrt{\Delta}}^{+1 / \sqrt{\Delta}} \varphi_{\Delta ; f}(y) \varphi_{\Delta ; f}(z) \delta\left(\Phi_{\Delta}{ }^{-1}(\xi)-F_{\Delta}(y, z)\right) d y d z,
$$

but it is probably best to follow through the thought process dictated by (4.5)-(4.7) the first few times.

At any rate, we are now seeking an $f \in L^{1}(\mathbb{R})$ which satisfies

$$
f=\mathscr{F}_{4}(f) \text {. }
$$

The principal result of this section can now be stated:

Theorem 4.1. Provided that $\Delta$ is sufficiently small, there is a unique one-parameter family of positive, symmetric $L^{1}$ functions, $f_{\Delta}$, with $\left\|f_{A}\right\|_{1}=1$, satisfying $E q$. (4.9). The family has the property that

$$
\lim _{\Delta \rightarrow 0} f_{\Delta}=\mathscr{G}^{\sigma^{*}}
$$

with $\mathscr{G}^{\sigma}$ a (normalized) Gaussian of width $\sigma^{2}$ :

$$
\mathscr{G}^{\sigma}(x)=\left(\pi \sigma^{2}\right)^{-1 / 2} \exp \left[-x^{2} / \sigma^{2}\right]
$$

and $\left(\sigma^{*}\right)^{2}=2 /\left[p_{G}\left(2 p_{G}-1\right)\right]$.

Theorem 4.1 implies the much desired:

Corollary. For $\Delta>0$ sufficiently small, there is a spin glass solution to the system (4.9) described by a density $\varrho_{\Delta}(x)$. The $\Delta \downarrow 0$ limit is singular; however, under the scaling $\varrho_{\Delta}(x) \rightarrow \sqrt{\Delta} \varrho_{\Delta}(x / \sqrt{\Delta})$, the limiting function is Gaussian with width $\left(\sigma^{*}\right)^{2}$.

\section{$I V b$. The Linear Operators and Their Eigenfunctions}

Despite the somewhat formidable appearance of $\mathscr{F}_{A}$, the operator is quite simple at $\Delta=0$. Indeed, here $\mathscr{F}_{\Delta}$ is just a rescaled convolution operator, and Eq. (4.9) reads

$$
f(x)=p_{G}{ }^{-1} \int f(y) f\left(x / p_{G}-y\right) d y, \quad p_{G} \equiv 1 / \sqrt{2},
$$


the solutions of which are the Gaussians, $\mathscr{G}^{\sigma}{ }_{0 .}$ (The reason for the 0 subscript will become clear shortly.)

Given a known solution to a functional equation, a natural tool is the implicit function theorem. Of course, in our case, this must fail at some stage since Proposition 3.9 assures us that positive solutions to (4.9) [or (3.4)] are not of arbitrary width. More explicitly, the discussion at the end of Sect. III demonstrates that only one of the solutions to (4.9) at $A=0$ can conceivably give rise to solutions when $p>p_{G}$.

Observe that Eq. (4.9) can be written as

$$
\mathscr{T}_{\Delta}(f)=B_{A}(f, f)-I(f),
$$

where $I$ is the identity operator and $B_{\Delta}$ is the bilinear operator

$$
B_{\Delta}(f ; g)[\xi]=\int_{-1 / \sqrt{\Delta}}^{+1 / \sqrt{\Delta}} \varphi_{\Delta ; f}(y) \varphi_{A ; g}(z) \delta\left(\Phi_{\Delta}^{-1}(\xi)-F_{\Delta}(y, z)\right) d y d z .
$$

Of the standard conditions under which the implicit function theorem is known to hold, the most important one is that the linear operator $\delta / \delta f \mathscr{T}_{\Delta}-$ assumed to exist - is invertible when evaluated at the "known solution." For our problem, the failure of this condition is a consequence of the following:

Proposition 4.2. For all $0 \leqq \Delta<1-1 / \sqrt{2}$ the Fréchet derivative $\delta / \delta f \mathscr{T}_{\Delta}(g ; \cdot)$ exists. Furthermore, if $L_{\Delta}{ }^{\sigma}$ is defined by $\delta / \delta f \mathscr{T}_{\Delta}\left(\mathscr{G}^{\sigma}{ }_{0} ; \cdot\right) \equiv L_{\Delta}{ }^{\sigma}-I$, then for all $\sigma>0$, the spectrum of $L_{0}{ }^{\sigma}$ consists of the points $\left(2 p_{G}{ }^{n} \mid n=0,1,2, \ldots\right)$. These points are eigenvalues and the associated eigenfunctions, denoted by $\mathscr{G}_{n}^{\sigma}(x)$, are the Hermite polynomials $H_{n}(x / \sigma)$ multiplied by $\mathscr{G}^{\sigma}{ }_{0}(x)$ :

$$
\mathscr{G}^{\sigma}{ }_{n}(x)=\sigma^{n}(-1)^{n} d^{n} / d x^{n} \mathscr{G}^{\sigma}{ }_{0}(x), \quad L_{A}{ }^{\sigma}\left(\mathscr{G}^{\sigma}{ }_{n}\right)=2 p_{G}{ }^{n} \mathscr{G}^{\sigma}{ }_{n} .
$$

Proof. Since $B_{A}$ is bilinear, it is easy to verify that for any $g \in L^{1}$, the Fréchet derivative of $\mathscr{T}_{A}$ at $g$ is

$$
\delta / \delta f \mathscr{T}_{\Delta}(g ; \eta)[\xi]=2 \int g(y) \eta(z) \delta\left(\Phi_{\Delta}{ }^{-1}(\xi)-F_{\Delta}(y, z)\right) d y d z-\eta(\xi) \equiv L_{\Delta}(g ; \eta)-\eta .
$$

As $\Delta \downarrow 0$, this becomes a linear convolution operator, so that, in particular,

$$
L_{0}=\delta /\left.\delta f \mathscr{F}_{\Delta}\left(\mathscr{G}^{\sigma}{ }_{0}\right)\right|_{\Delta=0}(\eta)=2 p_{G}{ }^{-1} \int \mathscr{G}^{\sigma}{ }_{0}\left(x / p_{G}-y\right) \eta(y) d y .
$$

The calculation of the spectrum of $L_{0}$ is a straightforward exercise which we will perform at unit width. Let $\mathscr{G}_{0} \equiv \mathscr{G}_{0}^{\sigma}$, and denote by $\widetilde{\mathscr{G}}_{0}$ the Fourier transform of $\mathscr{G}_{0}$. The (standard) Hermite polynomials are defined by the Rodrigues formula

$$
H_{n}(x)=\mathscr{G}_{0}{ }^{-1}(x)(-1)^{n} d^{n} / d x^{n} \mathscr{G}_{0}(x) .
$$

Now, we use the identity

$$
\mathscr{G}_{0}(k)=\left[\tilde{\mathscr{G}}_{0}\left(p_{G} k\right)\right]^{2}
$$

to deduce that

$$
\left[p_{G}{ }^{-1} \int \mathscr{G}_{0}\left(x / p_{G}-y\right) \mathscr{G}_{n}(y) d y\right]^{\sim}=\left(i k p_{G}\right)^{n}\left[\widetilde{G}_{0}\left(p_{G} k\right)\right]^{2}=\left(i k p_{G}\right)^{n} \widetilde{\mathscr{G}}_{0}(k) .
$$


Whence

$$
p_{G}{ }^{-1} \int \mathscr{G}_{0}\left(x / p_{G}-y\right) \mathscr{G}_{n}(y) d y=p_{G}{ }^{n} \mathscr{G}_{n}(x),
$$

as advertised.

It is easy to see that the above eigenvalues (and zero) exhaust the spectrum of $L_{0}{ }^{\sigma}$. Indeed, one can explicitly compute the resolvents $R_{\lambda}\left(L_{0}{ }^{\sigma}\right)$ and verify that they are bounded by a constant times the inverse of the distance to the nearest eigenvalue.

Corollary. For all $\sigma>0$, the linear operators $\delta /\left.\delta f \mathscr{T}_{\Delta}\right|_{\Delta=0}\left(\mathscr{G}^{\sigma}{ }_{0} ; \cdot\right) \equiv L_{0}{ }^{\sigma}-I$ are of index zero with $\operatorname{dim} \operatorname{ker}\left(L_{0}{ }^{\sigma}-I\right)=1$.

Proof. That $\mathscr{G}^{\sigma}{ }_{2}$ is the only nontrivial function in the kernel of $L_{0}{ }^{\sigma}-I$ is a consequence of the above calculations. Similar logic indicates that $\mathscr{G}^{\sigma}{ }_{2} \notin \operatorname{Ran}\left(L_{0}{ }^{\sigma}-I\right)$, but all else can be reached. Hence, dim coker $\left(L_{0}{ }^{\sigma}-I\right)=1$.

Thus the linear operator does not possess an inverse. This is a reexpression of something we knew from the probabilistic methods of the previous section; in the neighborhood of the critical point, the asymptotic behavior of this system is governed by its intrinsic nonlinearities.

Before we proceed, there are a few additional features of the linear operator $L_{0}{ }^{\sigma}$ and the bilinear operator $B_{0}$ which merit discussion.

1) While Proposition 4.2 is fresh in mind, the reader should verify [by a derivation identical to (4.15)-(4.18)] the formula

$$
B_{0}\left(\mathscr{G}_{n}^{\sigma} ; \mathscr{G}^{\sigma}{ }_{m}\right)=p_{G}{ }^{n+m} \mathscr{G}_{n+m}^{\sigma},
$$

which will be indispensible later.

2) Most of the eigenvalues of $L_{0}{ }^{\sigma}-I$ are less than zero, the three exceptions being $\mathscr{G}^{\sigma}{ }_{0}, \mathscr{G}^{\sigma}{ }_{1}$, and $\mathscr{G}^{\sigma}{ }_{2}$. Of course, $\mathscr{G}^{\sigma}{ }_{2}$ with eigenvalue 0 is a perturbative manifestation of the fact that Gaussians of all width satisfy (4.10). The eigenvalue of 1 for $\mathscr{G}^{\sigma}{ }_{0}$ stems from the fact that the bilinear operator $B_{\Delta}$ is "integral-preserving." By this we mean that if $f, g \in L^{1}$ then for any $\Delta$,

$$
\int B_{\Delta}(f, g)=\left(\int f\right)\left(\int g\right) \text {. }
$$

Thus, if one seeks a solution to (4.9) by starting with an initial function and iterating it indefinitely with $B_{\Delta}$, some caution must be taken to ensure that the initial function integrates to one. This "trivial" unstable mode is a consequence of the fact that $B_{A}$ is bilinear, and is easily remedied by a slight modification of the operator $\mathscr{F}_{\Delta}$ [see Eq. (5.15)]. Finally, there is $\mathscr{G}^{\sigma}{ }_{1}$ with eigenvalue $\sqrt{2}-1$. Of course, we are "not interested" in this function since we have already restricted ourselves to the even subspace of $L^{1}(\mathbb{R})$. Nonetheless, the presence of this odd function with positive eigenvalue is a reflection of the inherent instability of this system to ferromagnetic boundary conditions. The eigenvalue of $\mathscr{G}^{\sigma}{ }_{1}$ should be compared with the stability calculation of Proposition 3.1.

\section{IVc. Existence of Nontrivial Solutions}

According to the results of the previous subsection, we cannot make direct use of the implicit function theorem. However, in instances such as these, it does provide us with a "poor man's center manifold theorem:" 
Theorem 4.3. Let $\mathbb{X}, \mathbb{Y}$ denote Banach spaces and $\mathscr{T}_{A}: \mathbb{X} \rightarrow \mathbb{Y}$ be a $C^{2}$ map defined in some neighborhood of $\left(\Delta=0, f=f_{0}\right)$, satisfying $\mathscr{T}_{\Delta}\left(f_{0}\right)=0$. Furthermore, suppose with $\delta \mathscr{T}_{0} / \delta f \equiv \delta / \delta f \mathscr{T}_{0}\left(f_{0} ; \cdot\right)$ is a linear Fredholm operator with $\operatorname{dim} \operatorname{ker}\left(\delta \mathscr{T}_{0} / \delta f\right)<\infty$ and $\operatorname{dim} \operatorname{coker}\left(\delta \mathscr{T}_{0} / \delta f\right)<\infty$. If $\psi \in \operatorname{ker}\left(\delta \mathscr{T}_{0} / \delta f\right)$ and $\Delta$ is small enough, then for $\varepsilon$ sufficiently small, there is a function $g_{\varepsilon, \Delta}(\psi) \in \mathbb{X} \backslash \operatorname{ker}\left(\delta \mathscr{T}_{0} / \delta f\right)$ such that

$$
\mathscr{T}_{\Delta}\left(f_{0}+\varepsilon \psi+g_{\varepsilon, \Delta}\right) \in \operatorname{coker}\left(\delta \mathscr{T}_{0} / \delta f\right) .
$$

Moreover, for fixed $\psi, g_{\varepsilon, 4}$ is unique.

Proof. This is a standard result in bifurcation theory which has its roots in the original work of Liapunov and Schmitt. For a discussion of these and related matters see, for example, [35].

Remark. Since, in our case, $\operatorname{dim} \operatorname{ker}\left(\delta \mathscr{T}_{0} / \delta f\right)=\operatorname{dim} \operatorname{coker}\left(\delta \mathscr{T}_{0} / \delta f\right)=1$, the above theorem reduces our analysis to the case of "one equation, one unknown;" thus there is some reason for hope.

Let us pause to contrast our situation [here in $\left.L^{1}(\mathbb{R})\right]$ with the typical applications of the above theorem. It is often the case that, in the formalism of the statement of Theorem 4.3 , the vector $f_{0}$ continues to satisfy the equation $\mathscr{T}_{4}\left(f_{0}\right)=0$ in a neighborhood of $\Delta=0$; however, such a solution is deemed "undesirable" generally for reasons of stability. One is therefore seeking a new family of solutions bifurcating from $f_{0}$ which depend continuously on the parameter $\Delta$. It should be observed that back on $[-1,+1]$, where we are interested in probability measures, this is a reasonably fair description of our predicament. It is not, however, a complete description. Indeed, as evidenced by our gyrations on $L^{1}(\mathbb{R})$, the nature of the new solutions (should they exist) will be drastically different from the unstable solution, a point mass at 0 .

On $L^{1}(\mathbb{R})$, we differ from this "standard picture" in several ways. Most importantly, there are no "known solutions" - except at $\Delta=0$, and at $\Delta=0$, one might argue that there are too many! In fact, this surplus and deficiency compensate for each other quite nicely, as will shortly become clear.

Let us continue our discussion of the "standard picture" for the special case $\operatorname{dim} \operatorname{ker}\left(\delta_{\mathscr{T}_{0}} / \delta f\right)=\operatorname{dim} \operatorname{coker}\left(\delta \mathscr{T}_{0} / \delta f\right)=1$. Then, if $\mu$ is any nonzero linear functional which vanishes off of $\operatorname{coker}\left(\delta \mathscr{T}_{0} / \delta f\right)$, we may define

$$
h(\varepsilon, \Delta)=\mu\left(\mathscr{T}_{0}\left(f_{0}+\varepsilon \psi+g_{\varepsilon, \Delta}\right)\right) .
$$

Now, in the usual case, we have

$$
h(0,4) \equiv 0
$$

in a neighborhood of $\Delta=0$. It is also not unusual to find that

$$
\left\|g_{\varepsilon, 4}\right\|=o(\varepsilon) \text {. }
$$

Under these circumstances, the origin is a critical point $\left(\left.\nabla h\right|_{00}=0\right)$ and the Hessian matrix is nonpositive. If, in addition, one can guarantee that the cross derivative satisfies $h_{\varepsilon A} \neq 0$, then the origin is a saddle point, which implies the existence of a nontrivial level curve in the $\varepsilon \Delta$ plane starting from the origin (see Fig. 9). 
Fig. 9. The usual case

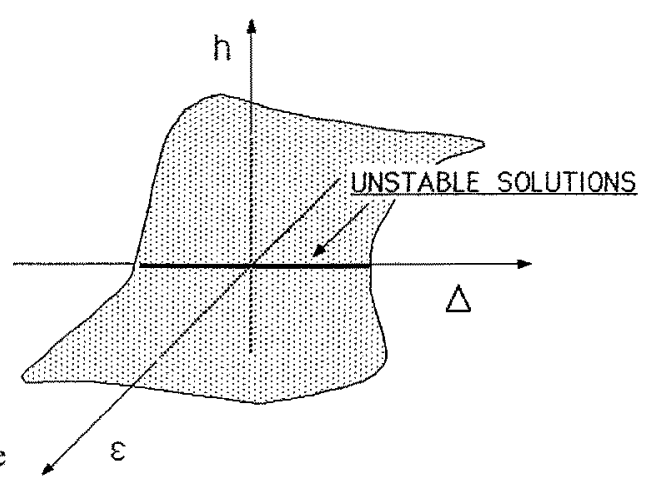

Now we have no knowledge of the behavior of our system for $\varepsilon=0, \Delta \neq 0$ indeed, our problem does not even make sense for $\Delta<0$. On the other hand, things are in remarkably good shape along the $\varepsilon$-axis. This will be demonstrated by an explicit construction of the (implicitly defined) function $g_{\varepsilon, \Delta}$ of Theorem 4.3 at $\Delta=0$.

Lemma 4.4. Let $g_{\varepsilon, \Delta}^{\sigma}$ denote the functions described in Theorem 4.3; that is

$$
\mathscr{T}_{A}\left(\mathscr{G}^{\sigma}{ }_{0}+\varepsilon \mathscr{G}^{\sigma}{ }_{2}+g^{\sigma}{ }_{\varepsilon, \Delta}\right)=h^{\sigma}(\varepsilon, \Delta) \mathscr{G}^{\sigma}{ }_{2} .
$$

Then, at $\Delta=0$,

$$
\begin{gathered}
\left\|g_{\varepsilon, 0}^{\sigma}\right\|=O\left(\varepsilon^{2}\right), \\
h(\varepsilon, 0) \equiv 0 \quad \text { for all } \quad|\varepsilon|<1 / 4 .
\end{gathered}
$$

Proof. An explicit calculation shows that

$$
g_{\varepsilon, 0}^{\sigma}=\sum_{n=2}^{\infty}(1 / n !) \varepsilon^{n} \mathscr{G}_{2 n}^{\sigma}
$$

(which converges uniformly for $|\varepsilon|<1 / 4$ ) satisfies

$$
\mathscr{F}_{0}\left(\mathscr{G}^{\sigma}{ }_{0}+\varepsilon^{\mathscr{G}^{\sigma}}{ }_{2}+g^{\sigma}{ }_{\varepsilon, 0}\right)=\mathscr{G}^{\sigma}{ }_{0}+\varepsilon^{\mathscr{G}^{\sigma}}{ }_{2}+g^{\sigma}{ }_{\varepsilon, 0} .
$$

This, together with the uniqueness clause of Theorem 4.3, implies i) and ii).

Remark. Two consequences of the above lemma are worthy of note:

1) While Theorem 4.3 only guarantees that $g_{\varepsilon, 4}^{\sigma} \in \mathbb{X} \backslash \operatorname{ker}\left(\delta \mathscr{T}_{0} / \delta f\right)$, so that in general $g_{\varepsilon, A}^{\sigma}$ may contain a piece proportional to $\mathscr{G}_{0}^{\sigma}$, the expansion (4.25) contains no such component. This is a reflection of the integral preserving property of $B_{\Delta}$, and holds even for nonzero $\Delta$.

2) Although Theorem 4.3 only provides us with an $f$ satisfying $\mathscr{T}_{\Delta}(f) \in \operatorname{ker}\left(\delta \mathscr{T}_{0} / \delta f\right)$, at $\Delta=0$ we find the stronger result that $\mathscr{T}_{0}(f)=0$. This should not represent a startling revelation; indeed, the $f$ so constructed is none other than the expansion of a different Gaussian $\mathscr{G}^{\sigma^{\prime}}{ }_{0}$ with $\sigma^{\prime}=\sigma[1+4 \varepsilon]^{1 / 2}$.

Thus in $L^{1}(\mathbb{R})$ we find ourselves a $90^{\circ}$ rotation away from the usual situation: Along the $\varepsilon$-axis is our analogue of a family of known solutions. However, these 
Fig. 10. Our case

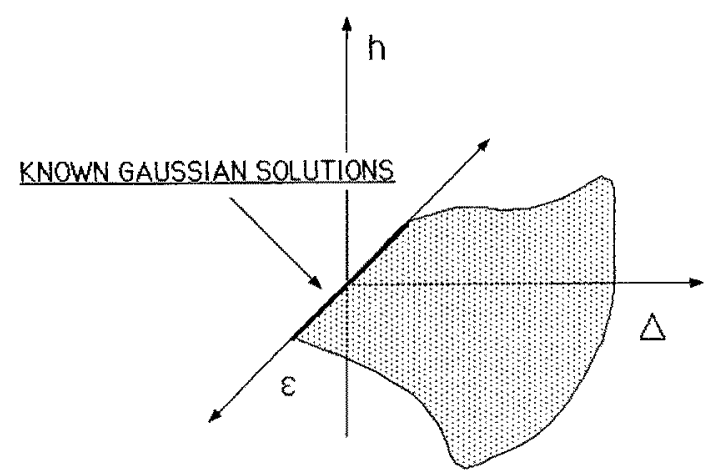

solutions do not correspond to a family of physical, unstable solutions; in some sense, they all represent the single critical solution.

With an eye for completing the proof of Theorem 4.1, let us summarize. The function $g_{\varepsilon, A}^{\sigma}$ is identically zero when $\Delta=0$ and $\varepsilon$ small enough. It is generically nonzero in a neighborhood of the origin; thus one can pretty much bank on the fact that the Hessian

$$
\left|h_{i j}^{\sigma}\right| \equiv h_{\varepsilon \varepsilon}^{\sigma} h_{\Delta A}^{\sigma}-h^{\sigma}{ }_{\varepsilon \Delta} h^{\sigma}{ }_{\Delta \varepsilon}
$$

will be negative when evaluated at zero. The nontrivial issue then is whether the origin is a critical point. It is at this stage that the condition of "the correct width" becomes manifest.

Lemma 4.5. Let $h^{\sigma}(\varepsilon, 4)$ be the function defined in $E q$. (4.24). Then the origin is a critical point for $h^{\sigma}$ iff $\sigma=\sigma^{*}=2 /\left[p_{G}\left(2 p_{G}{ }^{-1}\right)\right]$. Furthermore, for this value of the width, the origin is a saddle point.

Proof. By Lemma 4.4, for any width $\sigma, h_{\varepsilon}^{\sigma}(0,0) \equiv d /\left.d \varepsilon\left[h^{\sigma}(\varepsilon, 0)\right]\right|_{\varepsilon=0}=0$. Thus to verify that the origin is a critical point, we must show that

$$
h^{\sigma}{ }_{\Delta}(0,0) \equiv d /\left.d \Delta\left[h^{\sigma}(0, \Delta)\right]\right|_{\Delta=0}=0 .
$$

By the definition (4.24) of $h^{\sigma},(4.28)$ is equivalent to the statement that $d /\left.d \Delta\left[\mathscr{T}_{\Delta}\left(\mathscr{G}^{\sigma}{ }_{0}\right)\right]\right|_{\Delta=0}$ has no component along $\mathscr{G}^{\sigma}{ }_{2}$.

Let us then compute $d /\left.d \Delta\left[\mathscr{T}_{d}\left(\mathscr{G}_{0}^{\sigma}\right)\right]\right|_{\Delta=0}$. Our calculation is simplified by the fact that we have chosen $\Phi_{A}$ to be the identity in a neighborhood of the origin. This allows us to deal with the stretched operator $\mathscr{H}_{4}-I: L^{1}[-1 / \sqrt{\Delta},+1 / \sqrt{\Delta}]$ $\rightarrow L^{1}[-1 / \sqrt{\Delta},+1 / \sqrt{\Delta}]$, ignoring contributions from the endpoints. The stretched operator may be written in the same form as Eq. (4.1'):

$$
\mathscr{H}_{\Delta}(h)-I(h)[x]=-h(x)+\int_{-1 / \sqrt{\Delta}}^{+1 / \sqrt{4}} h(y) h\left(Q_{1}(x, y ; \Delta)\right) Q_{2}(x, y ; \Delta) d y,
$$

where

$$
\begin{aligned}
& Q_{1}(x, y ; \Delta)=(x-p y) /\left(p-\Delta m^{2} x y\right) \\
& Q_{2}(x, y ; \Delta)=p\left(1-\Delta m^{2} y^{2}\right) /\left(p-\Delta m^{2} x y\right)^{2}
\end{aligned}
$$


and

$$
\begin{aligned}
\Delta & =p-p_{G} ; \quad p_{G}=\sqrt{2}, \\
p & =p(\Delta)=p_{G}+\Delta, \\
m^{2} & =m^{2}(\Delta)=2 p(\Delta)-1=m_{G}{ }^{2}+2 \Delta ; \quad m_{G}{ }^{2}=2 p_{G}-1=\sqrt{2}-1 .
\end{aligned}
$$

A straightforward calculation now gives

$$
d /\left.d \Delta\left[\mathscr{T}_{\Delta}\left(\mathscr{G}^{\sigma}{ }_{0}\right)\right]\right|_{\Delta=0}=d /\left.d \Delta\left[\left(\mathscr{H}_{A}-I\right)\left(\mathscr{G}^{\sigma}{ }_{0}\right)\right]\right|_{\Delta=0} \equiv \mathscr{J}_{1}+\mathscr{J}_{2}
$$

with

$$
\begin{aligned}
\mathscr{J}_{1}= & \int \mathscr{G}^{\sigma}{ }_{0}\left(x / p_{G}-y\right) \mathscr{G}^{\sigma}{ }_{0}(y)\left\{p_{G}{ }^{-1}\left(2 m_{G}{ }^{2} x y-1-p_{G} m_{G}{ }^{2} y^{2}\right)\right\} d y / p_{G}, \\
\mathscr{J}_{2}= & -\int\left[\mathscr{G}_{0}^{\sigma}\left(x / p_{G}-y\right)\right]^{\prime} \mathscr{G}^{\sigma}{ }_{0}(y)\left\{p_{G}{ }^{-2}\left[p_{G} y+\left(x-p_{G} y\right)\left(1-m_{G}{ }^{2} x y\right)\right]\right\} d y / p_{G} \\
= & \int \mathscr{G}^{\sigma}{ }_{0}\left(x / p_{G}-y\right) \mathscr{G}^{\sigma}{ }_{0}(y) \\
& \times\left\{2 p_{G}{ }^{-2} \sigma^{-2}\left(x / p_{G}-y\right)\left[p_{G} y+\left(x-p_{G} y\right)\left(1-m_{G}{ }^{2} x y\right)\right]\right\} d y / p_{G} .
\end{aligned}
$$

Reexpressing the terms in brackets as functions of $y / \sigma$ and $\left(x / p_{G}-y\right) / \sigma$, these integrals become

$$
\begin{aligned}
\mathscr{J}_{1}= & \int \mathscr{G}_{0}{ }_{0}\left(x / p_{G}-y\right) \mathscr{G}^{\sigma}{ }_{0}(y) \\
& \times\left\{-p_{G}{ }^{-1}+m_{G}{ }^{2} \sigma^{2}\left[2\left(\left(x / p_{G}-y\right) / \sigma\right)(y / \sigma)+(y / \sigma)^{2}\right]\right\} d y / p_{G} \\
\mathscr{H}_{2}= & \int \mathscr{G}_{0}{ }_{0}\left(x / p_{G}-y\right) \mathscr{G}^{\sigma}{ }_{0}(y) 2\left\{p_{G}{ }^{-1}\left[\left(\left(x / p_{G}-y\right) / \sigma\right)(y / \sigma)+\left(\left(x / p_{G}-y\right) / \sigma\right)^{2}\right]\right. \\
& \left.-m_{G}{ }^{2} \sigma^{2}\left[\left(\left(x / p_{G}-y\right) / \sigma\right)^{3}(y / \sigma)+\left(\left(x / p_{G}-y\right) / \sigma\right)^{2}(y / \sigma)^{2}\right]\right\} d y / p_{G}
\end{aligned}
$$

Next we use the identities for Hermite polynomials (see e.g. [36]):

$$
\begin{aligned}
\xi & =(1 / 2) H_{1}(\xi), \\
\xi^{2} & =(1 / 2) H_{0}(\xi)+(1 / 4) H_{2}(\xi), \\
\xi^{3} & =(3 / 4) H_{1}(\xi)+(1 / 8) H_{3}(\xi),
\end{aligned}
$$

and the definition

$$
\mathscr{G}^{\sigma}{ }_{n}(\xi)=\mathscr{G}^{\sigma}{ }_{0}(\xi) H_{n}(\xi / \sigma)
$$

to rewrite $\mathscr{J}_{1}$ and $\mathscr{J}_{2}$ as convolutions of the eigenfunctions. These are, however, not just any convolutions. Indeed, they may be expressed in terms of our bilinear operator $B_{0}$ :

$$
\begin{aligned}
\mathscr{J}_{1}+\mathscr{J}_{2}= & -p_{G}{ }^{-1} B_{0}\left[\mathscr{G}_{0}^{\sigma} ; \mathscr{G}^{\sigma}{ }_{0}\right]+2\left(m_{G}{ }^{2} \sigma^{2}+p_{G}{ }^{-1}\right) B_{0}\left[(1 / 2) \mathscr{G}^{\sigma}{ }_{1} ;(1 / 2) \mathscr{G}^{\sigma}{ }_{1}\right] \\
& +m_{G}{ }^{2} \sigma^{2} B_{0}\left[\mathscr{G}_{0}^{\sigma} ;(1 / 2) \mathscr{G}_{0}{ }_{0}+(1 / 4) \mathscr{G}^{\sigma}{ }_{2}\right] \\
& +2 p_{G}{ }^{-1} B_{0}\left[(1 / 2) \mathscr{G}^{\sigma}{ }_{0}+(1 / 4) \mathscr{G}^{\sigma}{ }_{2} ; \mathscr{G}^{\sigma}{ }_{0}\right] \\
& -2 m_{G}{ }^{2} \sigma^{2} B_{0}\left[(3 / 4) \mathscr{G}^{\sigma}{ }_{1}+(1 / 8) \mathscr{G}^{\sigma}{ }_{3} ;(1 / 2) \mathscr{G}^{\sigma}{ }_{1}\right] \\
& \left.-2 m_{G}{ }^{2} \sigma^{2} B_{0}\left[(1 / 2) \mathscr{G}^{\sigma}{ }_{0}+{ }^{(1} / 4\right) \mathscr{G}^{\sigma}{ }_{2} ;(1 / 2) \mathscr{G}^{\sigma}{ }_{0}+(1 / 4) \mathscr{G}^{\sigma}{ }_{2}\right]
\end{aligned}
$$


Evaluating the terms above via the convolution identity (4.19), it is found that

$$
d /\left.d \Delta\left[\mathscr{T}_{\Delta}\left(\mathscr{G}^{\sigma}{ }_{0}\right)\right]\right|_{\Delta=0}=\left[-(1 / 2) m_{G}{ }^{2} \sigma^{2}+p_{G}{ }^{-1}\right] p_{G}{ }^{2} \mathscr{G}_{2}^{\sigma}-(1 / 4) m_{G}{ }^{2} \sigma^{2} p_{G}{ }^{4} \mathscr{G}^{\sigma}{ }_{4} .
$$

Observe that for any $\sigma$, the above expression has no component along $\mathscr{G}^{\sigma}{ }_{0}$, consistent with the same property already noted for $g_{\varepsilon, \Delta}^{\sigma}$. However, the component along $\mathscr{G}_{2}{ }_{2}$ vanishes only for the special width $\sigma^{*}$ given by

$$
\left(\sigma^{*}\right)^{2}=2 / p_{G} m_{G}{ }^{2} .
$$

The reader should note that (4.39) compares favorably with the asymptotics of the stable band calculated in Sect. III.

Next we must verify that the origin is a saddle point for $h^{\sigma^{*}}$. This amounts to checking that the Hessian of Eq. (4.27) is negative at $\sigma=\sigma^{*}$ when $(\varepsilon, \Delta)=(0,0)$. Again, by Lemma 4.4 , for any width $\sigma, h^{\sigma}{ }_{\varepsilon \varepsilon}(0,0) \equiv d^{2} /\left.d \varepsilon^{2}[h(\varepsilon, 0)]\right|_{\varepsilon=0}=0$. Thus it suffices to show that

$$
h_{\varepsilon \Delta}^{\sigma}(0,0)=h_{\Delta \varepsilon}^{\sigma}(0,0) \equiv d^{2} /\left.d \varepsilon d \Delta\left[h^{\sigma}(\varepsilon, \Delta)\right]\right|_{\varepsilon=\Delta=0} \neq 0
$$

at $\sigma=\sigma^{*}$. But by Eq. (4.24), $h^{\sigma}{ }_{\varepsilon A}(0,0)$ is the component of $d /\left.d \Delta\left[(\delta / \delta f) \mathscr{T}_{\Delta}\left(\mathscr{G}^{\sigma}{ }_{0} ; \mathscr{G}^{\sigma}\right)\right]\right|_{\Delta=0}$ along $\mathscr{G}^{\sigma}{ }_{2}$. In other words, (4.40) is equivalent to the statement that the mixed derivative operator, when evaluated at the known solution $\mathscr{G}^{\sigma}{ }_{0}$ at $\Delta=0$, operating on an element $\mathscr{G}_{2}^{\sigma}$ of the kernel of the linear operator, must have some component along the cokernel $\mathscr{G}^{\sigma}{ }_{2}$.

In order to compute the mixed derivative on $\mathscr{G}^{\sigma}{ }_{2}$, we first take the functional derivative evaluated at $\mathscr{G}_{0}^{\sigma}$ :

$$
(\delta / \delta f) \mathscr{T}_{\Delta}\left(\mathscr{G}^{\sigma}{ }_{0} ; \mathscr{G}^{\sigma}{ }_{2}\right)=-I\left(\mathscr{G}_{2}^{\sigma}\right)+2 B_{\Delta}\left(\mathscr{G}^{\sigma}{ }_{0} ; \mathscr{G}^{\sigma}{ }_{2}\right) .
$$

The calculation of the derivative with respect to $\Delta$ is again simplified by our choice of $\Phi_{\Delta}$, which allows us to deal with the stretched form of the bilinear operator (i.e., the bilinear analogue of $\mathscr{H}_{A}$ ). The result is

$$
d /\left.d \Delta\left[(\delta / \delta f) \mathscr{T}_{\Delta}\left(\mathscr{G}_{0}^{\sigma} ; \mathscr{G}^{\sigma}{ }_{2}\right)\right]\right|_{A=0}=2\left[\overline{\mathscr{J}}_{1}\left(\mathscr{G}_{0}^{\sigma} ; \mathscr{G}^{\sigma}{ }_{2}\right)+\overline{\mathscr{J}}_{2}\left(\mathscr{G}_{0}^{\sigma} ; \mathscr{G}_{2}^{\sigma}\right)\right]
$$

where $\overline{\mathscr{J}}_{1}\left(\mathscr{G}_{0}^{\sigma} ; \mathscr{G}^{\sigma}{ }_{2}\right)$ and $\overline{\mathscr{J}}_{2}\left(\mathscr{G}^{\sigma}{ }_{0} ; \mathscr{G}^{\sigma}{ }_{2}\right)$ are the bilinear analogues of the integrals $\mathscr{J}_{1}$ and $\mathscr{J}_{2}$ of Eq. (4.31) in which the factors of $\mathscr{G}^{\sigma}{ }_{0}(y)$ are replaced by $\mathscr{G}^{\sigma}{ }_{2}(y)$ [e.g., $\left.\mathscr{J}_{1}=\overline{\mathscr{J}}_{1}\left(\mathscr{G}_{0}^{\sigma} ; \mathscr{G}^{\sigma}{ }_{0}\right)\right]$.

Next, we use the Hermite polynomial identity (4.34) to reexpress $\mathscr{G}^{\sigma}{ }_{2}(y)$ in the form

$$
\mathscr{G}_{2}^{\sigma}(y)=2\left(2 y^{2} / \sigma^{2}-1\right) \mathscr{G}^{\sigma}{ }_{0}(y)
$$

We then have

$$
d /\left.d \Delta\left[(\delta / \delta f) \mathscr{T}_{\Delta}\left(\mathscr{G}_{0}^{\sigma} ; \mathscr{G}_{2}{ }_{2}\right)\right]\right|_{\Delta=0}=4\left[2\left(\hat{\mathscr{F}}_{1}+\hat{\mathscr{F}}_{2}\right)-\left(\mathscr{J}_{1}+\mathscr{J}_{2}\right)\right],
$$

where $\hat{\mathscr{J}}_{1}$ and $\hat{\mathscr{J}}_{2}$ are analogues of $\mathscr{J}_{1}$ and $\mathscr{J}_{2}$ [see Eq. (4.31)], in which the integrands contain additional factors of $(y / \sigma)^{2}$. In order to evaluate these integrals, we again use identities (4.33)-(4.35), and the additional formula

$$
\xi^{4}=(3 / 4) H_{0}(\xi)+(3 / 4) H_{2}(\xi)+(1 / 16) H_{4}(\xi)
$$


to obtain

$$
\begin{aligned}
& \hat{\mathscr{J}}_{1}+\hat{\mathscr{J}}_{2}=-p_{G}{ }^{-1} B_{0}\left[\mathscr{G}_{0}^{\sigma} ;(1 / 2) \mathscr{G}^{\sigma}{ }_{0}+(1 / 4) \mathscr{G}^{\sigma}{ }_{2}\right] \\
& +2\left(m_{G}{ }^{2} \sigma^{2}+p_{G}{ }^{-1}\right) B_{0}\left[(1 / 2) \mathscr{G}^{\sigma}{ }_{1} ;(3 / 4) \mathscr{G}^{\sigma}{ }_{1}+(1 / 8) \mathscr{G}^{\sigma}{ }_{3}\right] \\
& +m_{G}{ }^{2} \sigma^{2} B_{0}\left[\mathscr{G}^{\sigma}{ }_{0} ;(3 / 4) \mathscr{G}^{\sigma}{ }_{0}+(3 / 4) \mathscr{G}^{\sigma}{ }_{2}+(1 / 16) \mathscr{G}^{\sigma}{ }_{4}\right] \\
& +2 p_{G}{ }^{-1} B_{0}\left[(1 / 2) \mathscr{G}^{\sigma}{ }_{0}+(1 / 4) \mathscr{G}^{\sigma}{ }_{2} ;(1 / 2) \mathscr{G}^{\sigma}{ }_{0}+(1 / 4) \mathscr{G}^{\sigma}{ }_{2}\right] \\
& -2 m_{G}{ }^{2} \sigma^{2} B_{0}\left[(3 / 4) \mathscr{G}^{\sigma}{ }_{1}+(1 / 8) \mathscr{G}^{\sigma}{ }_{3} ;(3 / 4) \mathscr{G}^{\sigma}{ }_{1}+(1 / 8) \mathscr{G}^{\sigma}{ }_{3}\right] \\
& -2 m_{G}{ }^{2} \sigma^{2} B_{0}\left[(1 / 2) \mathscr{G}^{\sigma}{ }_{0}+(1 / 4) \mathscr{G}^{\sigma}{ }_{2} ;(3 / 4) \mathscr{G}^{\sigma}{ }_{0}+(3 / 4) \mathscr{G}^{\sigma}{ }_{2}+(1 / 16) \mathscr{G}^{\sigma}{ }_{4}\right] \\
& =\left[p_{G}{ }^{-1}-(3 / 4) m_{G}{ }^{2} \sigma^{2}\right] p_{G}{ }^{2} \mathscr{G}_{2}^{\sigma}+\left[(1 / 4) p_{G}{ }^{-1}-(5 / 8) m_{G}{ }^{2} \sigma^{2}\right] p_{G}{ }^{4} \mathscr{G}_{4}{ }_{4} \\
& -(1 / 16) m_{G}{ }^{2} \sigma^{2} p_{G}{ }^{6} \mathscr{G}^{\sigma}{ }_{6} \text {. }
\end{aligned}
$$

Since the $\mathscr{G}^{\sigma}{ }_{2}$ component of $\mathscr{J}_{1}+\mathscr{I}_{2}$ vanishes for the special width $\sigma=\sigma^{*}$, it suffices [by Eq. (4.44)] to verify that the $\mathscr{G}^{\sigma}{ }_{2}$ component of $\hat{\mathscr{J}}_{1}+\hat{\mathscr{J}}_{2}$ is nonzero for $\sigma=\sigma^{*}$. This, however, is obvious:

$$
p_{G}{ }^{-1}-(3 / 4) m_{G}{ }^{2}\left(\sigma^{*}\right)^{2}=p_{G}{ }^{-1}[1-(3 / 4)(2)]=-(1 / 2) p_{G}{ }^{-1} \neq 0 \text {. }
$$

Thus, we have established that the origin is a saddle point for $h^{\sigma^{*}}$, which completes the proof of Lemma 4.5.

For future reference, let us just note that, for general width $\sigma$, the value of the cross derivative is

$$
\begin{aligned}
d / d & \left.\Delta\left[(\delta / \delta f) \mathscr{T}_{\Delta}\left(\mathscr{G}^{\sigma}{ }_{0} ; \mathscr{G}^{\sigma}\right)\right]\right|_{\Delta=0} \\
& =4\left\{\left[p_{G}{ }^{-1}-m_{G}{ }^{2} \sigma^{2}\right] p_{G}{ }^{2} \mathscr{G}^{\sigma}{ }_{2}+\left[(1 / 2) p_{G}{ }^{-1}-m_{G}{ }^{2} \sigma^{2}\right] p_{G}{ }^{4} \mathscr{G}^{\sigma}{ }_{4}-(1 / 8) m_{G}{ }^{2} \sigma^{2} p_{G}{ }^{6} \mathscr{G}_{6}{ }_{6}\right\} \\
& \equiv-\kappa_{2} \mathscr{G}^{\sigma}{ }_{2}-\kappa_{4} \mathscr{G}^{\sigma}{ }_{4}-\kappa_{6} \mathscr{G}^{\sigma}{ }_{6} .
\end{aligned}
$$

As was already apparent from Eqs. (4.38) and (4.46), this has no component along $\mathscr{G}^{\sigma}{ }_{0}$, which is again a perturbative manifestation of the integral-preserving property (4.20).

The previous lemma shows that only the Gaussian of the "correct" width, $\left(\sigma^{*}\right)^{2}=2 / p_{G} m_{G}{ }^{2}$, has a chance of bifurcating into a family of new solutions at nonzero $\Delta$. That the origin is a saddle point of $h^{\sigma^{*}}$ shows that this family does indeed bifurcate. Thus we have

Corollary. Provided that $\Delta$ is sufficiently small, there is a unique one-parameter family of symmetric $L^{1}$ functions, $f_{\Delta}$, which satisfy the equation

$$
f_{\Delta}=\mathscr{F}_{\Delta}\left(f_{\Delta}\right) \text { a.e. }
$$

\section{IVd. Positivity of the Solutions}

The previous subsection establishes the existence of nontrivial solutions to $f_{A}=\mathscr{F}_{\Delta}\left(f_{A}\right)$. This is practically the result we want, except that, as of yet, we have no guarantee that the $f_{\Delta}$ satisfying the above are positive. Although positivity should be obvious on moral grounds, if looked at in the wrong light, such questions can seem unreasonably difficult to analyze. The resolution, however, turns out to be straightforward. 
Proposition 4.6. The fixed point equation

$$
f_{\Delta}=\mathscr{F}_{\Delta}\left(\left|f_{\Delta}\right|\right)
$$

also admits a unique one-parameter family of symmetric $L^{1}$ solutions when $\Delta$ is small enough.

Proof. This is established by checking that all the linear operators associated with the new problem are identical to the objects computed previously. Now this may seem unlikely, since taking absolute values can easily lead to singularities in derivatives. However, the result is a consequence of the fact that the derivative of the absolute value of a function which is known to be positive equals the derivative of the function itself. From this point of view, it is essential to our analysis that the "point" in $L^{1}$ where all these derivatives are taken is $\mathscr{G}^{\sigma}{ }_{0}$, a function which is uniformly positive on any bounded subset of $\mathbb{R}$.

Rather than attempting to demonstrate the above principle in full generality, we will be content with a single example: Let us consider the Fréchet derivative $(\delta / \delta f) B_{0}\left(\left|\mathscr{G}^{\sigma}{ }_{0}\right| ;\left|\mathscr{G}^{\sigma}{ }_{0}\right|\right)$. Pick $\eta \in C^{\infty}{ }_{0}$ with $\|\eta\|_{1}=1$. The derivative is evaluated via the limit

$$
\lim _{\varepsilon \rightarrow 0}(1 / \varepsilon)\left[B_{0}\left(\left|\mathscr{G}^{\sigma}{ }_{0}+\varepsilon \eta\right| ;\left|\mathscr{G}^{\sigma}{ }_{0}+\varepsilon \eta\right|\right)-B_{0}\left(\left|\mathscr{G}^{\sigma}{ }_{0}\right| ;\left|\mathscr{G}^{\sigma}{ }_{0}\right|\right)\right] .
$$

Here, the argument is trivial. For $\varepsilon$ sufficiently small, the function $\mathscr{G}^{\sigma}{ }_{0}(x)+\varepsilon \eta(x)$ is positive $\forall x \in \mathbb{R}$. Thus we may omit the absolute value bars in the above expression. This is all we need since $C^{\infty}{ }_{0}$ is dense in $L^{1}$. However, since the result seems rather surprising, let us make the proof somewhat more explicit:

Let $f \in L^{1}$, with $\|f\|_{1}=1$, and for general $\psi(x)$, denote by $\psi^{-}$the negative part of $\psi$, i.e. $\psi^{-}(x)=|\psi(x)|$ if $\psi(x) \leqq 0$ and zero otherwise. Now

so that

$$
\left|\mathscr{G}^{\sigma}{ }_{0}+\varepsilon f\right|=\mathscr{G}^{\sigma}{ }_{0}+\varepsilon f+2\left[\mathscr{G}_{0}^{\sigma}+\left.\varepsilon f\right|^{-},\right.
$$

$$
\begin{aligned}
B_{\Delta}\left(\left|\mathscr{G}^{\sigma}{ }_{0}+\varepsilon f\right| ;\left|\mathscr{G}^{\sigma}{ }_{0}+\varepsilon f\right|\right)= & B_{A}\left(\mathscr{G}_{0}^{\sigma}{ }_{0} \mathscr{G}_{0}{ }_{0}\right)+2 \varepsilon B_{A}\left(\mathscr{G}_{0}{ }_{0} ; f\right) \\
& \left.+4 B_{\Delta}\left(\mathscr{G}_{0}^{\sigma}{ }_{0} ; \mathscr{G}^{\sigma}{ }_{0}+\varepsilon f\right]^{-}\right) \\
& +B_{\Delta}\left(\varepsilon f+2\left[\mathscr{G}^{\sigma}{ }_{0}+\varepsilon f\right]^{-} ; \varepsilon f+2\left[\mathscr{G}^{\sigma}{ }_{0}+\varepsilon f\right]^{-}\right) .
\end{aligned}
$$

Observing that

$$
\left|\varepsilon f+2\left[\mathscr{G}^{\sigma}{ }_{0}+\varepsilon f\right]^{-}\right| \leqq|\varepsilon f|+2\left|\left[\mathscr{G}_{0}^{\sigma}+\varepsilon f\right]^{-}\right| \leqq 3|\varepsilon f|,
$$

the last term in Eq. (4.51) is seen to have an $L^{1}$ norm which is $O\left(\varepsilon^{2}\right)$. The first two terms evolve into the Fréchet derivative.

It remains to show that

$$
0=\lim _{\varepsilon \rightarrow 0}(1 / \varepsilon)\left\|B_{A}\left(\mathscr{G}_{0}^{\sigma} ;\left[\mathscr{G}_{0}^{\sigma}+\varepsilon f\right]^{-}\right)\right\|_{1} .
$$

By the property (4.20), the above is just

$$
\lim _{\varepsilon \rightarrow 0}(1 / \varepsilon) \int\left[\mathscr{G}_{0}^{\sigma}+\varepsilon f\right]^{-\cdots} d x .
$$

Next, write $f^{-}(x)=f_{N}{ }^{-}(x)+r_{N}(x)$ with $f_{N}^{-}(x)=f^{-}(x)$ if $|x| \leqq N$ and $f^{-}(x) \leqq N$, and zero otherwise. It is easily seen that

$$
\left[\mathscr{G}^{\sigma}{ }_{0}+\varepsilon f\right]^{-}=\left[\mathscr{G}^{\sigma}{ }_{0}-\varepsilon f_{N}\right]^{-}+\left[\mathscr{G}^{\sigma}{ }_{0}-\varepsilon r_{N}\right]^{-},
$$


and that

$$
\left[\mathscr{G}^{\sigma}{ }_{0}-\varepsilon f_{N}\right]^{-} \equiv 0
$$

for $\varepsilon$ small enough. Thus $\forall N$

$$
\lim _{\varepsilon \rightarrow 0}(1 / \varepsilon)\left\|B_{\Delta}\left(\mathscr{G}_{0}^{\sigma} ;\left[\mathscr{G}^{\sigma}{ }_{0}+\varepsilon f\right]^{-}\right)\right\|_{1} \leqq \int r_{N} d x,
$$

which can be made as small as desired by taking $N$ large enough.

The calculations of the other linear operators - including "cross derivatives" are analogous.

Proof of Theorem 4.1. For small values of $\Delta$, we have established the existence of solutions to two separate equations: $f_{\Delta}^{(n)}$ satisfies

while $f_{\Delta}^{(p)}$ satisfies

$$
f_{A}^{(u)}=\mathscr{F}\left(f_{A}^{(u)}\right),
$$

$$
f_{\Delta}^{(p)}=\mathscr{F}\left(\mid f_{\Delta}^{(p)}\right) \text {. }
$$

It remains to show that $f_{\Delta}{ }^{(u)}=f_{A}{ }^{(p)} \equiv f_{A}$ and that $\left\|f_{A}\right\|_{1}=1$. To this end, observe that $f_{A}{ }^{(p)}$ is automatically positive, since the output of $\mathscr{F}_{A}$ on a positive function is positive. This implies that

$$
\int f_{A}^{(p)}=\left\|f_{\Delta}^{(p)}\right\|_{1} .
$$

However, by the integral-preserving property (4.20) [and by Eq. (4.59)], this implies $\left\|f_{\Delta}^{(p)}\right\|_{1}=1$. We have also inadvertently shown that $f_{\Delta}^{(p)}$ satisfies Eq. (4.58); thus by uniqueness of solutions, $f_{A}^{(p)}=f_{A}^{(u)}$.

\section{Properties of the Solution}

Having established the existence of a spin glass solution to Eq. (2.27)-in its various guises - it is worthwhile to investigate some of its basic properties. The analysis in this section is by no means exhaustive; indeed, full justice to this problem would be the contents of a separate paper.

We will therefore be content with studying three aspects of the solution:

1) It follows from the results of the previous section that the scaled solution is a Gaussian of width $\sigma^{*}=\left[2 / p_{G}\left(2 p_{G}-1\right)\right]^{1 / 2}$ at the critical point $\Delta=0$. As we move away from the critical point, one might imagine two types of corrections. First, there may be a correction to the width of the solution; basically, this would mean that the solution looks like a Gaussian of a different width $\sigma(\Delta)=\sigma^{*}[1+\delta \sigma(\Delta)]$. Second, there may be deviations from Gaussian behavior so that the solution goes like $\mathscr{G}^{\sigma(\Delta)}[1+\delta f(\Delta)]$, with $\delta f(\Delta)$ an explicitly non-Gaussian function. In Subsect. Va, we will compute these corrections and show that they are of the same order.

2) In Subsect. $\mathrm{Vb}$, we examine stability of the solution by calculating the eigenvalues of (the linear approximation to) the functional derivative $\delta / \delta f \mathscr{T}_{\Delta}\left(f_{\Delta} ; \cdot\right)$. It is found that (except for the trivial "integral-preserving mode") all eigenvalues are negative to first order in $\Delta$, consistent with stability. From the considerations of Sect. IV, it should be clear that the only potentially dangerous eigenvalue is that of the "width mode," for which we also calculate the lowest order correction to the eigenfunction.

3) In Subsect. Ve, we examine the asymptotic behavior of the solution near the tails of the distribution, i.e. when the single-site magnetizations are near those of the 
deterministic ferromagnetic system. Here we find a truly nonperturbative effect: The probability that the single-site magnetizations of the spin glass are within $\zeta$ of $m^{(\infty)}(p)$ vanishes faster than $\exp \left[-\zeta^{-A(p)}\right]$, where $A(p)$ depends explicitly on the temperature parameter $p=\tanh \left(1 / k_{B} T\right)$. We also find that the probability is bounded above by an expression of the same form with a closely related function $B(p)$. In light of this (temperature-dependent) "singular" behavior, it is reassuring to know that a solution exists.

\section{Va. The Leading Non-Gaussian Correction}

As should be clear from the lesson of Lemma 4.4 [cf. Eq. (4.25)], expanding the solution $f_{A}$ in the form

$$
f_{\Delta}=\sum_{n} c_{n} \mathscr{G}^{\sigma^{*}}
$$

and starting at the $c_{n}$ does not immediately allow one to draw conclusions about the departure of $f_{\Delta}$ from a Gaussian. Indeed, one first must decide from which Gaussian $f_{A}$ is supposed to be departing. Our criterion (which is by no means the only one) is to find a width $\sigma(\Delta)$ such that the expansion of $f_{A}$ in Hermite functions of width $\sigma(\Delta)$ has vanishing component along $\mathscr{G}^{\sigma(\Delta)}{ }_{2}$.

It turns out that many of the properties of the $f_{4}(x)$ satisfying (4.9) can be studied using the fact that $f_{A}$ is related to the probability density of the random variables satisfying (3.4). Our strategy will be to take Eq. (3.4) and rescale it by the unknown with $\sigma$. Thus we have

related by

$$
\begin{aligned}
X_{\sigma} & \equiv X / \sigma, \\
Y_{\sigma} & \equiv Y / \sigma, \\
Z_{\sigma} & \equiv Z / \sigma,
\end{aligned}
$$

$$
X_{\sigma}=p\left[Y_{\sigma}+Z_{\sigma}\right] /\left[1+m^{2} \sigma^{2} Y_{\sigma} Z\right] \text {. }
$$

It is clear that if we choose $\sigma(4)$ by requiring that

$$
E\left[H_{2}\left(X_{\sigma}\right)\right]=0,
$$

then $f_{\Delta}\left(\Phi_{\sigma^{2}}(x)\right)$ will have no component along $\mathscr{G}^{\sigma}{ }_{2}$. A similar result then holds for $f_{\Delta}(x)$ to all orders in perturbation theory (i.e., the result is perturbatively independent of the decompactification). This is enough to satisfy us for the time being since we can only treat (5.3) order by order in $\Delta$.

It turns out that the convenient quantity to calculate is $m^{2} \sigma^{2}$, and that the computationally useful perturbation parameter is

$$
\delta \equiv\left(2 p^{2}-1\right) / p^{2}=\left(4 / p_{G}\right) \Delta-12 \Delta^{2}+O\left(\Delta^{3}\right)
$$

rather than $\Delta$. In terms of this, the result is

Proposition 5.1. The width satisfying (5.4) is given by

$$
m^{2} \sigma^{2}=(1 / 2) \delta[1+(9 / 8) \delta]+o\left(\delta^{2}\right) .
$$

Remark. By (5.5), the leading order term in $m^{2} \sigma^{2}$ is $(1 / 2) \delta=\left(2 / p_{G}\right) \Delta+O\left(\Delta^{2}\right)$, which the reader should recognize as $m^{2} \sigma^{* 2}+O\left(A^{2}\right)$. 
Proof. Using the identity (4.34) and the distributional equation (5.3), we have

$$
\begin{aligned}
H_{2}\left(X_{\sigma}\right)= & 4 p^{2}\left[Y_{\sigma}+Z_{\sigma}\right]^{2} /\left[1+m^{2} \sigma^{2} Y_{\sigma} Z_{\sigma}\right]^{2}-2 \\
& =p^{2}\left\{4 Y_{\sigma}{ }^{2}+4 Z_{\sigma}{ }^{2}-16 m^{2} \sigma^{2} Y_{\sigma}{ }^{2} Z_{\sigma}{ }^{2}+12 m^{4} \sigma^{4} Y_{\sigma}{ }^{2} Z_{\sigma}{ }^{2}\left(Y_{\sigma}{ }^{2}+Z_{\sigma}{ }^{2}\right)\right\} \\
& -2+\Theta_{\text {odd }}\left(Y_{\sigma}, Z_{\sigma}\right)+R_{\sigma}\left(Y_{\sigma}, Z_{\sigma}\right),
\end{aligned}
$$

where $\Theta_{\text {odd }}\left(Y_{\sigma}, Z_{\sigma}\right)$ has vanishing expectation due to symmetry, and $R_{\sigma}$ is the remainder. Using the advance knowledge (demonstrated in Sect. IV) that $\sigma^{2}=O(\Delta)$, it can be shown that

$$
E\left[\left|R_{\sigma}\right|\right]=O\left(m^{2} \sigma^{2}\right)^{3} .
$$

Organizing the right-hand side of (5.6) into Hermite functions, a little effort shows that

$$
\begin{aligned}
H_{2}\left(X_{\sigma}\right)= & p^{2}\left\{H_{2}\left(Y_{\sigma}\right)+H_{2}\left(Z_{\sigma}\right)+4-m^{2} \sigma^{2}\left[H_{2}\left(Y_{\sigma}\right)+2\right]\left[H_{2}\left(Z_{\sigma}\right)+2\right]\right. \\
& +3 m^{4} \sigma^{4}\left[(1 / 16) H_{4}\left(Y_{\sigma}\right)+(3 / 4) H_{2}\left(Y_{\sigma}\right)+(3 / 4)\right]\left[H_{2}\left(Z_{\sigma}\right)+2\right] \\
& \left.+3 m^{4} \sigma^{4}\left[(1 / 16) H_{4}\left(Y_{\sigma}\right)+(3 / 4) H_{2}\left(Y_{\sigma}\right)+(3 / 4)\right]\left[H_{2}\left(Z_{\sigma}\right)+2\right]\right\} \\
& -2+\Theta_{\text {odd }}\left(Y_{\sigma}, Z_{\sigma}\right)+R_{\sigma}\left(Y_{\sigma}, Z_{\sigma}\right) .
\end{aligned}
$$

Choosing $\sigma$ such that $E\left[H_{2}\left(X_{\sigma}\right)\right]=0$, we find

$$
0=4 p^{2}-2-4 p^{2} m^{2} \sigma^{2}-9 p^{2} m^{4} \sigma^{4}+o(4)^{2} .
$$

It should be remarked that in the above, we have again used knowledge from Sect. IV, this time of the fact that

$$
E\left[H_{4}\left(X_{\sigma}\right)\right]=o(1)
$$

(although we will shortly replace this with an even stronger statement). Solving (5.9) to second order in $\delta$ reproduces the statement of the proposition.

To obtain the first non-Gaussian correction, we look at higher powers of (5.3) and perform similar heroics.

Proposition 5.2. If $\sigma$ is chosen according to Proposition 5.1, then

$$
\tilde{\alpha} \equiv E\left[H_{4}\left(X_{\sigma}\right)\right]=-24 \delta+o\left(\delta^{2}\right) .
$$

Proof. We use the well-known identity

$$
\mathrm{H}_{4}(\xi)=16 \xi^{4}-12-12 \mathrm{H}_{2}(\xi)
$$

on the random variable $X_{\sigma}$. Thus, we need an expression for $16 X_{\sigma}{ }^{4}$ in terms of $Y_{\sigma}$ and $Z_{\sigma}$ which is reliable to order $\sigma^{2}$. The result is

$$
\begin{aligned}
16 X_{\sigma}{ }^{4}= & p^{4}\left\{H_{4}\left(Y_{\sigma}\right)+12 H_{2}\left(Y_{\sigma}\right)+H_{4}\left(Z_{\sigma}\right)+12 H_{2}\left(Z_{\sigma}\right)+24\right. \\
& +6\left(H_{2}\left(Y_{\sigma}\right)+2\right)\left(H_{2}\left(Z_{\sigma}\right)+2\right) \\
& -4 m^{2} \sigma^{2}\left[\left(H_{4}\left(Y_{\sigma}\right)+12 H_{2}\left(Y_{\sigma}\right)+12\right)\left(H_{2}\left(Z_{\sigma}\right)+2\right)\right. \\
& \left.\left.+\left(H_{4}\left(Z_{\sigma}\right)+12 H_{2}\left(Z_{\sigma}\right)+12\right)\left(H_{2}\left(Y_{\sigma}\right)+2\right)\right]\right\} \\
& +\Theta_{\text {odd }}^{\prime}\left(Y_{\sigma}, Z_{\sigma}\right)+R_{\sigma}^{\prime}\left(Y_{\sigma}, Z_{\sigma}\right) .
\end{aligned}
$$


Subtracting $12 \mathrm{H}_{2}\left(\mathrm{X}_{\sigma}\right)+12$ from the right-hand side of (5.12), we get a (reliable) expression for $\mathrm{H}_{4}\left(\mathrm{X}_{\sigma}\right)$. Averaging both sides, we obtain

$$
\tilde{\alpha}\left[1-2 p^{2}\right]=16 p^{4}\left(3-12 m^{2} \sigma^{2}\right)-12+o\left[\left(\sigma^{2}\right)^{2}\right],
$$

which, after a little thought, is seen to imply

$$
\tilde{\alpha}=-24 \delta+o\left(\delta^{2}\right) .
$$

Combining the two results of this section and using (5.5), we have

Proposition 5.3. The perturbative approximation to the solution to Eq. (4.9) is given, to leading order, by

$$
f_{\Delta}=\mathscr{G}^{\sigma(\Delta)}{ }_{0}+\alpha(\Delta) \mathscr{G}^{\sigma(\Delta)}{ }_{4}+o(\Delta)
$$

with

$$
m^{2} \sigma^{2}(\Delta)=\left(2 / p_{G}\right) \Delta+12 \Delta^{2}+o\left(\Delta^{2}\right), \quad \alpha(\Delta)=\tilde{\alpha} /\left(2^{4} 4 !\right)=-\left(1 / 4 p_{G}\right) \Delta+o(\Delta) .
$$

\section{Vb. Linear Stability and Perturbations in the Width Mode}

The moment analysis of Sect. III, together with the existence of a solution to (4.9), suggest that the solution is of the attractive type. We do not, as of yet, have any strong results in this direction. We can, however, show that the linear operator approximating the functional derivative $\delta / \delta f \mathscr{T}_{\Delta}\left(f_{\Delta} ; \cdot\right)$ has only negative eigenvalues; this is, of course, a necessary condition for establishing more complete statements concerning the stability of solutions to (4.9).

Remark. One should mention that any time there is a solution to $f_{A}=\mathscr{F}_{A}\left(f_{A}\right)$, the operator $\delta / \delta f \mathscr{T}_{\Delta}\left(f_{\Delta} ; \cdot\right)$ always has $f_{A}$ as an eigenfunction of eigenvalue unity. This corresponds to the "trivial" growth mode, and can easily be killed by redefining the functional. For example, using

$$
\mathscr{F}_{\Delta}(f) \equiv\|f\|^{-3 / 2} \mathscr{F}_{\Delta}(f)
$$

does not affect our analysis in the slightest, and $\mathscr{F}_{A}^{\prime}$ is kind enough to lower the eigenvalue of $f_{\Delta}$ by $3 / 2$.

Since the major danger is the second eigenvalue (i.e., the eigenvalue of the "width mode") which vanishes at the critical point, we will examine this mode in some detail.

Proposition 5.4. For $\Delta$ sufficiently small, all eigenvalues (except the maximum) of the linear approximation to $\delta / \delta f_{T_{A}}\left(f_{A} ; \cdot\right)$ are negative. Explicitly, these eigenvalues are given by

$$
\lambda_{n}=-1+2 p^{n}\left[1-n(n-1) p_{G}{ }^{-1} \Delta\right] .
$$

Furthermore, to lowest order in $\Delta$, the second eigenfunction is given by

$$
f^{(2)}{ }_{\Delta}=\mathscr{G}^{\sigma(\Delta)}{ }_{2}+\beta(\Delta) \mathscr{G}^{\sigma(\Delta)}{ }_{4}+\gamma(\Delta) \mathscr{G}^{\sigma(\Delta)}{ }_{6}+o(\Delta),
$$

where $\sigma(\Delta)$ is given in Proposition 5.3, and

$$
\beta(\Delta)=-3 p_{G}^{-1} \Delta, \quad \gamma(\Delta)=-(1 / 4) p_{G}{ }^{-1} \Delta .
$$


Proof. First, let us determine the linear approximation to $\delta / \delta f \mathscr{T}_{A}\left(f_{A} ; \cdot\right)$. To do this, we recall that $\delta / \delta f \mathscr{F}_{A}$ is just twice the bilinear operator $B_{A}$ of Eq. (4.12), and expand the latter in the form

$$
B_{\Delta}(\cdot ; \cdot)=B_{0}(\cdot ; \cdot)+\Delta B_{1}(\cdot ; \cdot)+R_{\Delta}(\cdot ; \cdot) .
$$

Here $\left\|R_{\Delta}(\cdot ; \cdot)\right\|=o(\Delta)$, and $\Delta B_{1}(\cdot ; \cdot)$ is just half of the "cross-derivative operator" considered in Lemma 4.5. Now, using the results of Proposition 5.3, we may write the solution $f^{(0)}{ }_{A}$ in the form

$$
f^{(0)}{ }_{\Delta}=\mathscr{G}^{\sigma(\Delta)}{ }_{0}+\alpha(\Delta) \mathscr{G}^{\sigma(\Delta)}{ }_{4}+r^{(0)}{ }_{\Delta}
$$

where $\sigma(\Delta)$ and $\alpha(\Delta)$ are given in the statement of Proposition 5.3, and $\left\|r^{(0)}{ }_{\Delta}\right\|=o(\Delta)$. The linear approximation is therefore

$$
\begin{aligned}
\delta / \delta f \mathscr{T}_{\Delta}\left(f^{(0)}{ }_{\Delta} ; \cdot\right)= & -I(\cdot)+2 B_{0}\left(\mathscr{G}^{\sigma(\Delta)}{ }_{0} ; \cdot\right)+2 \alpha B_{0}\left(\mathscr{G}^{\sigma(\Delta)}{ }_{4} ; \cdot\right) \\
& +2 \Delta B_{1}\left(\mathscr{G}^{\sigma(\Delta)}{ }_{0} ; \cdot\right)+R_{\Delta}^{\prime}(\cdot)
\end{aligned}
$$

with $\left\|R_{\Delta}^{\prime}(\cdot)\right\|=o(\Delta)$.

As indicated above, our principal concern is the eigenfunction $f^{(2)}{ }_{\Delta}$ with the property $f^{(2)}{ }_{4} \rightarrow \mathscr{G}_{2}{ }^{\sigma^{*}}$ as $\Delta \downarrow 0$. Let us construct $f^{(2)}{ }_{\Delta}$ perturbatively. Thus, we write

$$
f^{(2)}{ }_{\Delta}=\mathscr{G}^{\sigma(\Delta)}{ }_{2}+\beta(\Delta) \mathscr{G}^{\sigma(\Delta)}{ }_{4}+\gamma(\Delta) \mathscr{G}^{\sigma(\Delta)}{ }_{6}+r^{(2)}{ }_{\Delta} \text {, }
$$

where, presumably, $\left\|r^{(2)}{ }_{\Delta}\right\|=o(\Delta)$, while $\beta$ and $\gamma$ are $O(\Delta)$. To determine the coefficients $\beta$ and $\gamma$, and the eigenvalue, we must solve

$$
\delta / \delta f \mathscr{T}_{A}\left(f^{(0)}{ }_{A} ; f^{(2)}{ }_{A}\right)=\lambda_{2} f^{(2)}{ }_{A}
$$

to leading order in 4 . Our calculation is tremendously simplified by the fact that we have already computed $2 B_{1}\left(\mathscr{G}_{0}^{\sigma} ; \mathscr{G}^{\sigma}\right)$ in the proof of Lemma 4.5. Explicitly, from Eq. (4.48), we have

$$
2 B_{1}\left(\mathscr{G}_{0}^{\sigma} ; \mathscr{G}^{\sigma}{ }_{2}\right)=-\left[\kappa_{2} \mathscr{G}^{\sigma}{ }_{2}+\kappa_{4} \mathscr{G}^{\sigma}+\kappa_{6} \mathscr{G}_{6}^{\sigma}\right]
$$

where, for $\sigma=\sigma^{*}$,

$$
\begin{aligned}
& \kappa_{2}=\left(4 / p_{G}\right) p_{G}{ }^{2}, \\
& \kappa_{4}=\left(6 / p_{G}\right) p_{G}{ }^{4}, \\
& \kappa_{6}=\left(1 / p_{G}\right) p_{G}{ }^{6} .
\end{aligned}
$$

Plugging (5.18) and (5.19) and solving to lowest order, we find

$$
\begin{aligned}
\lambda_{2} & =-\Delta \kappa_{2}=-2 p_{G}{ }^{-1} \Delta, \\
\beta & =-2 \Delta \kappa_{4}=-3 p_{G}{ }^{-1} \Delta, \\
\gamma & =-(4 / 3)\left[\Delta \kappa_{6}-2 \alpha p_{G}{ }^{6}\right]=-(1 / 4) p_{G}{ }^{-1} \Delta .
\end{aligned}
$$

Thus the eigenvalue $\lambda_{2}$ is negative, consistent with stability.

It remains to check the other eigenvalues. Since we are not interested in the perturbed eigenfunctions, we need only check the diagonal part of the operator in (5.18). The only nontrivial calculation is to compute the coefficient of $\mathscr{G}_{n}$ in $2 B_{1}\left(\mathscr{G}_{0}^{\sigma} ; \mathscr{G}^{\sigma}{ }_{n}\right)$. Omitting the details, the result is

$$
\mu_{n}=-2 p_{G}{ }^{n}\left[n(n-1) / p_{G}\right] A,
$$


which gives the shift in the eigenvalues of (the linear approximation to) the linear operator. Combining this with the zero order terms from (5.18), we obtain

$$
\lambda_{n}=-1+2 p^{n}\left[1-n(n-1) p_{G}{ }^{-1} \Delta\right] .
$$

Thus, all eigenvalues in the symmetric subspace (save the trivial maximum one) are negative, as asserted.

\section{Vc. Asymptotics at the Tails}

In the preceding subsection, as well as in most of the previous analysis, we have been concerned with the stochastic behavior of the system when our random variables are near the center of the distribution. By rescaling - in one form or another - this has allowed perturbative control of certain aspects of the problem.

There is a fundamentally nonperturbative feature in this system which no amount of rescaling can get around: The random variables described by Eq. (3.4) live on $[-1,+1]$. Thus perturbation theory has to break down at some order (depending on $\Delta$ ), because the probability of observing magnetizations with absolute value approaching $m^{(\infty)}(p)$ must vanish.

In this subsection, we will use some elementary probabilistic methods to estimate the behavior of the empirical distribution of random variables satisfying Eq. (3.4). Our principal result is:

Theorem 5.5. Let $X$ denote the random variable satisfying Eq. (3.4) and distributed according to a density. Define $\mathbf{G}(\zeta)=\operatorname{Prob}(X>1-\zeta)$. Then for $\zeta$ sufficiently small,

$$
\mathbf{G}(\zeta) \leqq \exp \left[-1 / \zeta^{a}\right],
$$

b)

$$
\mathbf{G}(\zeta) \geqq \exp \left[-1 / \zeta^{b}\right]
$$

whenever

$$
a>A(p) \equiv(\log 2) /[\log [(1-p) /(2 p)] \mid, \quad b<B(p) \equiv(\log 2) /[\log [(1-p) / p] \mid .
$$

Remark. These estimates indicate that the functions $\varrho_{A}(x)$ are "highly nonanalytic" at $|x|=1$. Of course, this sentence is meaningless since we have no guarantee of analyticity properties for smaller values of $x$. Nonetheless, the above theorem is indicative of the global nature of the solutions to Eq. (3.4).

Proof. The first observation [necessary for both the bounds a) and b)] is that for all $Y, Z$ in $(-1,+1), F(Y, Z)$ is increasing in both arguments. This is easily checked by computing

$$
\partial F / \partial Z=p\left(1-m^{2} Y^{2}\right) /\left(1+m^{2} Y Z\right)^{2},
$$

with a similar result for $\partial F / \partial Y$.

Now let $0<\zeta<1$, and express $X$, distributionally, in terms of the independent random variables $Y$ and $Z$ according to Eq. (3.4a). Due to the increasing property, it is seen that even if $Z=1, X$ cannot exceed $1-\zeta$ unless $Y$ exceeds a certain value, which we denote by $1-u(\zeta)$. Thus $u(\zeta)$ is defined by

$$
F(1-u(\zeta), 1)=1-\zeta
$$


Solving for $u(\zeta)$, we find

$$
u(\zeta)=2 p \zeta /\left(1-p+\zeta m^{2}\right) .
$$

Again making use of the increasing property, it is clear that both $Y$ and $Z$ must exceed $1-u(\zeta)$ in order that $X$ exceed $1-\zeta$. This implies the inequality

$$
\mathbf{G}(\zeta) \leqq \mathbf{G}^{2}(u(\zeta)) \text {. }
$$

It turns out that the remainder of the derivation is much cleaner if we reverse the emphasis of (5.31), i.e. we write

with

$$
\mathbf{G}(\zeta(u)) \leqq \mathbf{G}^{2}(u)
$$

$$
\zeta(u)=(1-p) u /\left(2 p-m^{2} u\right) .
$$

Pick any value $u_{0}<1$, and define the sequence $u_{1}=\zeta\left(u_{0}\right), u_{2}=\zeta\left(u_{1}\right)$, etc. Equation (5.32) tells us that

$$
G\left(u_{n}\right) \leqq G\left(u_{0}\right)^{2^{n}} .
$$

We now claim that $u_{n} \sim[(1-p) /(2 p)]^{n}$, in the sense that

$$
\lim _{n \rightarrow \infty} u_{n}^{1 / n}=(1-p) /(2 p) \text {. }
$$

To see this, observe that $\zeta(u) \geqq u(1-p) /(2 p)$, which implies that $\forall n$, $u_{n} \geqq u_{0}[(1-p) /(2 p)]^{n}$, giving a bound one way. On the other hand, the $u_{n}$ are strictly decreasing, which means that $\forall k$

$$
u_{n+k} \leqq u_{k}\left[(1-p) /\left(2 p-m^{2} u_{k}\right)\right]^{n},
$$

from which an opposite bound is obtained.

We are thus permitted to write $2^{n} \sim u_{n}{ }^{-A(p)}$ with

$$
A(p)=(\log 2) /|\log [(1-p) /(2 p)]| .
$$

Then by choosing any $a<A$, the bound is established at the points $\zeta=u_{n}$ for $n$ large enough. But by the monotonicity of $\mathbf{G}(\zeta)$, this implies that $\mathbf{G}(\zeta) \leqq \exp \left[-1 / \zeta^{a}\right]$ in the intervals $u_{n+1}<\zeta \leqq u_{n}$. Since we have been generous with our estimate of the power (i.e., we refrained from choosing $a=A$ ), we can now find an $n_{0}$, so that the result holds whenever $\zeta \leqq u_{n_{0}}$.

The second result follows in an analogous fashion. Letting $\zeta \in(0,1)$ we will find $a v(\zeta)$ such that if both $Y$ and $Z$ exceed $1-v(\zeta), X$ is forced to exceed $1-\zeta$. It is easy to verify that

$$
\zeta(v)=v\left[2(1-p)+m^{2} v\right] /\left[2 p-m^{2}\left(2 u-u^{2}\right)\right] .
$$

Repeating the argument which established the upper bound - remembering to be generous enough to choose $b>B$ - the second result is seen to hold for $\zeta$ sufficiently small.

Remark. It is easily seen that improved upper bounds can be obtained by the incorporation of more sophisticated input conditions. The lower bounds cannot be improved, in the sense that under the assumption that $\mathbf{G}(\zeta) \sim \exp \left[-1 / \zeta^{c}\right]$, it is easily shown that $c=B(p)$. 
Acknowledgements. The authors are grateful to R. Durrett, J, Guckenheimer, H. Kesten, M. Randeria, and D. Rokhsar for their comments, suggestions, and encouragement.

\section{References}

1. Sherrington, D., Kirkpatrick, S.: Solvable model of a spin glass. Phys. Rev. Lett. 35, 1792 (1975)

2. Thouless, D.J.: Spin-glass on a Bethe lattice. Phys. Rev. Lett. 56, 1082 (1986)

3. Omari, R., Prejéan, J.J., Souletie, J.: Critical measurements in the spin glass CuMn. J. Physique 44, 1069 (1983)

Omari, R., Prejéan, J.J., Souletie, J.: In Heidelberg Colloquium on Spin Glasses. van Hemmen, J.L., Morgenstern, I. (eds.), pp. 70-78. Berlin, Heidelberg, New York: Springer 1983

4. Bouchiat, H., Monod, P.: Critical analysis of the nonlinear magnetization in the AgMn spin glass, preprint (1985) to appear in J. Mag. and Mag. Mat.

5. Edwards, S.F., Anderson, P.J.: J. Phys. F 5, 965 (1975)

6. van Enter, A.C.D., Griffiths, R.B.: The order parameter in a spin glass. Commun. Math. Phys. 90, $319(1983)$

7. Thouless, D.J., Anderson, P.W., Palmer, R.G.: Solution of a "Solvable model of a spin glass." Philos. Mag. 35, 593 (1977)

8. Ueno, Y., Oguchi, T.: Random ordered phase characteristic of quenched mixtures of Ising spins. J. Phys. Soc. Japan 40, 1513 (1976)

Oguchi, T., Ueno, Y.: Statistical theory of the random ordered phase in quenched bond mixtures. J. Phys. Soc. Japan 41, 1123 (1976)

Oguchi, T., Ueno, Y.: Prog. Theor. Phys. 57, 683 (1977)

Oguchi, T., Takano, F.: J. Mag., Mag. Mat. 31-34, 1301 (1983)

9. Katsura, S., Fujiki, S., Inawashiro, S.: Spin-glass phase in the site Ising model. J. Phys. C 12, 2839 (1979)

Katsura, S.: Entropy of the spin-glass state in the binary mixture of the ferro- and antiferromagnetic random Ising model at $T=0$. Physica 104A, $333(1980)$

Fujiki, S., Abe, Y, Katsura, S.: Computer Phys. Commun. 25, 119 (1982)

10. Anderson, P.W.: In: Ill-condensed matter. Les Houches Session XXXI. Balian, R., Maynard, R., Toulouse, G. (eds.), pp. 159-262. Amsterdam; North-Holland 1979

11. de Almeida, J.R.L., Thouless, D.J.: J. Phys. A 11, 983 (1978)

12. Parisi, G.: Toward a mean field theory for spin glasses. Phys. Lett. A 73, 203 (1979)

Parisi, G.: Infinite number of order parameters for spin-glasses. Phys. Rev, Lett. 43, 1754 (1979)

Parisi, G.: J. Phys. A 13, L115 (1980)

Parisi, G.: J. Phys. A 13, 1101 (1980)

Parisi, G.: J. Phys. A 13, 1807 (1980)

13. de Dominicis, C., Young, A.P.: Weighted averages and order parameters for the infinite range Ising spin glass. J. Phys. A 16, 2063 (1983)

14. Parisi, G.: Order parameter for spin glasses. Phys. Rev. Lett. 50, 1946 (1983)

15. Houghton, A., Jain, S., Young, A.P.: Role of initial conditions in spin glass dynamics and significance of Parisi's $q(x)$. J. Phys. C 16, L375 (1983)

16. Young, A.P.: Direct determination of the probability distribution for the spin-glass order parameter. Phys. Rev. Lett. 51, 1206 (1983)

17. Mézard, M., Parisi, G., Sourlas, N., Toulouse, G., Virasoro, M.: Phys. Rev. Lett. 52, 1186 (1984)

18. Sompolinsky, H., Zippelius, A.: Dynamic theory of the spin-glass phase. Phys. Rev. Lett. 47, 359 (1981)

Sompolinsky, H.: Time-dependent order parameters in spin-glasses. Phys. Rev. Lett. 47, 935 (1981)

Sompolinsky, H., Zippelius, A.: Relaxational dynamics of the Edwards-Anderson model and the mean-field theory of spin-glasses. Phys. Rev. B 25, 6860 (1982) 
19. Bhatt, R.N., Young, A.P.: Phys. Rev. Lett. 54, 924 (1985)

20. Ogielski, A.T., Morgenstern, I.: Phys. Rev. Lett. 54, 928 (1985)

21. McMillan, W.L.: Phys. Rev. B 31, 340 (1985)

22. Bray, A.J., Moore, M.A.: Phys. Rev. B 31, 631 (1985)

23. Griffiths, R.B.: Nonanalytic behavior above the critical point in a random Ising ferromagnet. Phys. Rev. Lett. 23, 17 (1969)

24. Randeria, M., Sethna, J.P., Palmer, R.G.: Phys. Rev. Lett. 54, 1321 (1985)

25. Fisher, D.S., Huse, D.A.: The ordered phase of short-range Ising spin glasses, preprint (1986)

26. Bowman, D.R., Levin, K.: Spin-glass theory in the Bethe approximation: Insights and problems. Phys. Rev. B 25, 3438 (1982)

27. Bruinsma, R.: Random-field Ising model on a Bethe lattice. Phys. Rev. B 30, 289 (1984)

28. Baxter, RJ.: Exactly solved models in statistical mechanics. New York: Academic Press 1982, Chap. 4

29. Wu, F.Y.: The Potts model. Rev. Mod. Phys. 54, 235 (1982)

30. Peruggi, F., di Liberto, F., Monroy, G.: The Potts model on Bethe lattices: I. General results. J. Phys. A 16, 811 (1983)

31. Fisher, M.E., Essam, J.W.: J. Math. Phys. 2, 609 (1961)

32. Fortuin, C.M., Kasteleyn, P.W.: On the random-cluster model. I. Introduction and relation to other models. Physica 57, 536 (1972)

33. Harris, T.E.: Proc. Camb. Phil. Soc. 56, 13 (1960)

34. Fortuin, C.M., Kasteleyn, P.W., Ginibre, J.: Correlation inequalities on some partially ordered sets. Commun. Math. Phys. 22, 89 (1981)

35. Berger, M.S.: Nonlinearity and functional analysis: Lectures on nonlinear problems in mathematical analysis. New York: Academic Press 1977, Chap. 4.1

36. Abramowitz, M., Stegun, I.A. (eds.): Handbook of mathematical functions. New York: Dover Publications, ninth printing, p. 801

Communicated by A. Jaffe

Received April 17, 1986 